Marquette University

e-Publications@Marquette

Master's Theses (2009 -)

Dissertations, Theses, and Professional Projects

\title{
Personalized Pain Study Platform Using Evidence- Based Continuous Learning Tool
}

Amit Kumar Saha

Marquette University

Recommended Citation

Saha, Amit Kumar, "Personalized Pain Study Platform Using Evidence-Based Continuous Learning Tool" (2018). Master's Theses (2009-). 512.

https://epublications.marquette.edu/theses_open/512 


\title{
PERSONALIZED PAIN STUDY PLATFORM USING EVIDENCE-BASED CONTINUOUS \\ LEARNING TOOL
}

\author{
by
}

Amit Kumar Saha, B.Sc

A Thesis submitted to the Faculty of the Graduate School,

Marquette University,

in Partial Fulfillment of the Requirements for the Degree of Master of Science.

Milwaukee, Wisconsin

December 2018 


\section{ABSTRACT \\ PERSONALIZED PAIN STUDY PLATFORM USING \\ EVIDENCE-BASED CONTINUOUS \\ LEARNING TOOL}

Amit Kumar Saha, B.Sc.

Marquette University, 2018

With the increased accessibility to mobile technologies, research utilizing mobile technologies in medical and public health area has also increased. The efficiency and effectiveness of healthcare services are also improved by introduction of mobile technologies. Effective pain treatment requires regular and continuous pain assessment of the patients. Mobile Health or mHealth has been an active interdisciplinary research area for more than a decade to research pain assessment through different software research tools. Different mHealth support systems are developed to assess pain level of patient using different techniques. Close attention to participant's self- reported pain along with data mining based pain level detection could help the healthcare industry and researchers to deliver effective health services in pain treatment. Pain expression recognition can be a good way for data mining based approach though pain expression recognition itself may utilize different approach based on the research study scope. Most of the pain research tools are study or disease specific. Some of the tools are pain specific (lumber pain, cancer pain etc) and some are patient group specific (neonatal, adult, woman etc). This results in recurrent but potentially avoidable costs such as time, money, and workforce to develop similar service or software research tools for each research study. Based on the pain study research characteristics, it is possible to design and implement a customizable and extensible generic pain research tool. In this thesis, we have proposed, designed, and implemented a customizable personalized pain study platform tool following a micro service architecture. It has most of the common software research modules that are needed for a pain research study. These include real-time data collection, research participant management, role based access control, research data anonymization etc. This software research tool is also used to investigate pain level detection accuracy using evidence-based continuous learning from facial expression which yielded about $71 \%$ classification accuracy. This tool is also HIPAA compliant and platform independent which makes it device independent, privacy-aware, and security-aware. 


\title{
ACKNOWLEDGEMENTS
}

\author{
Amit Kumar Saha, B.Sc
}

I would like to thank my wife, Anindita Saha, for her unparalleled support, inspiration and guidance. Without her, it would not be possible to achieve this. I would like to pay my gratitude to my friends and family especially my father and mother for their inspiration. I would also like to thank my advisor Dr. Sheikh Iqbal Ahamed for his guidance over the entire research period, as well as his ability to push me to go beyond what I thought possible. I would like to thank the other members of my committee, Dr. Praveen Madiraju, and Dr. Shion Guha, for their advice and support. Last but not least, I would also like to thank Dr. Md Osman Gani for his feedback and continuous supervisory help during the research. Finally, I would like to thank my colleagues of Ubicomp lab for their support. 
ACKNOWLEDGEMENTS .......................................... i

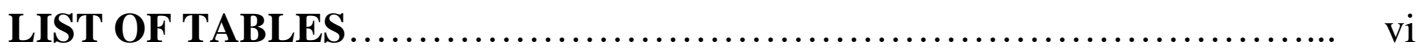

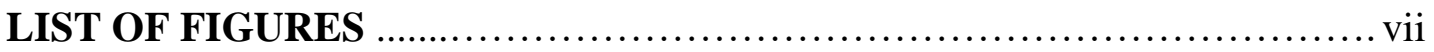

CHAPTER 1: INTRODUCTION ..................................... 1

1.1 BACKGROUND.......................................... 1

1.2 OBJECTIVES............................................ 2

1.3 THESIS ORGANIZATION ............................... 2

CHAPTER 2: RELATED STUDY ................................... 4

2.1 PAIN ASSESSMENT TOOLS .......................... 5

CHAPTER 3: PLATFORM REQUIREMENT ANALYSIS ................ 8

3.1 MOBILE APPLICATION …............................ 8

3.2 SERVER APPLICATION ................................. 9

3.3 USER ENROLLMENT ….............................. 9

3.3.1 RESEARCHERS ................................. 10

3.3.2 PARTICIPANT ...................................... 10

3.4 PATIENT HISTORY MANAGEMENT ..................... 10 
5.1 DEVELOPMENT PROCESS AND REQUIREMENTS

ANALYSIS

5.3.1 AILING USER'S POINT OF VIEW

5.3.2 RESEARCHER'S POINT OF VIEW

5.6.1 ROLE-BASED ACCESS 
6.2 SYSTEM COMMUNICATION ........................... 30

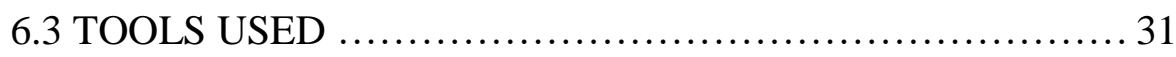

6.4 SERVER CONFIGURATION .......................... 33

6.5 SYSTEM DESCRIPTION ............................. 34

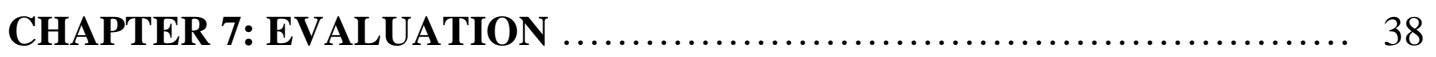

7.1 PLATFORM EVALUATION ........................... 38

7.2 PAIN DETECTION TOOL EVALUATION ................ 40

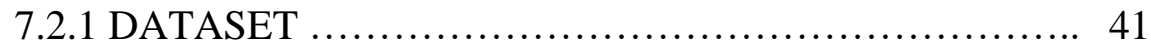

7.2.2 CROSS-SECTIONAL EVALUATION ................. 41

7.2.3 LONGITUDINAL EVALUATION .................... 43

7.2.4 DEMOGRAPHIC EVALUATION ................... 45

7.2.5 RESULTS ..................................... 45

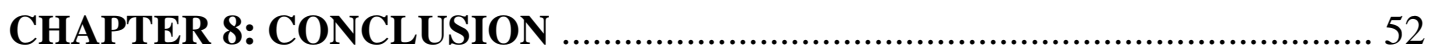

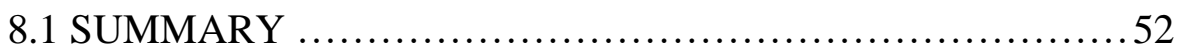

8.2 INTELLECTUAL MERIT ................................ 52

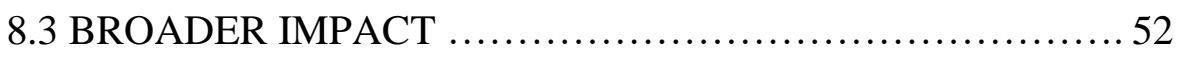

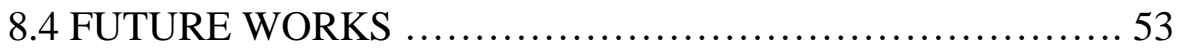


Bibliography 


\section{LIST OF TABLES}

$1 \quad$ Existing Pain Assessment Tools .................................... 6

2 Numeric Score Based Pain Scale Systems ........................... 7

3 Common Platform Architectures and Styles............................ 21

$4 \quad$ Eight Quality Characteristics of ISO/IEC 25010:2011_................ 39

$5 \quad$ Platform Evaluation based on ISO/IEC 25010:2011 ................... 40

6 Mean Absolute Error for 10-fold cross validation......................... 46

7 Classification Accuracy of Experiment-1 for 10-fold cross validation...... 48

$8 \quad$ Cross-sectional Demographic Evaluation................................ 49

9 Classification Accuracy of Experiment-2 for a 10-fold cross validation.... 50 


\section{LIST OF FIGURES}

$1 \quad$ Face Transformation ..................................................... 13

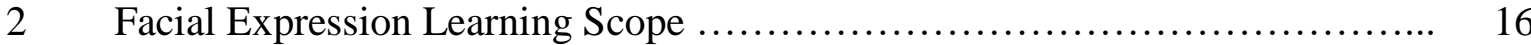

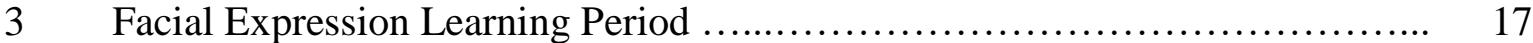

$4 \quad$ Personalized Training ..................................................... 18

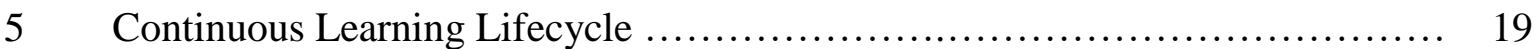

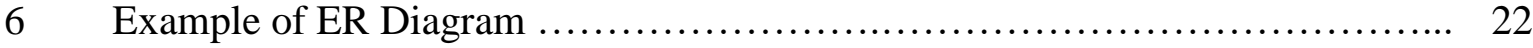

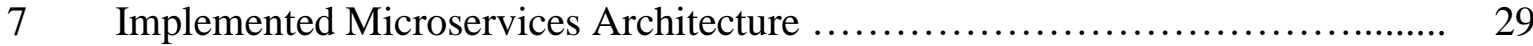

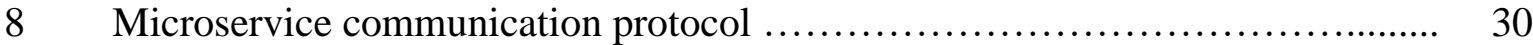

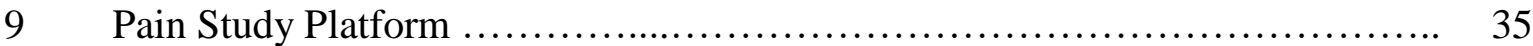

10 Research-Researcher Relationship ........................................ 35

11 Research-Researcher Module Screenshots ................................. 36

12 Patient Enrollment Module Screenshots................................... 37

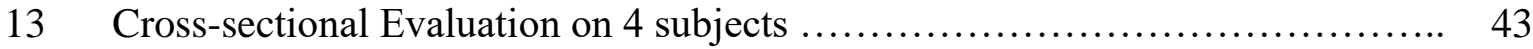

14 Longitudinal Evaluation on 4 subjects ..................................... 45

15 Class-distribution for Experiment -1 in Longitudinal Dataset .................. 49

16 Class-distribution for Experiment -2 in Longitudinal Dataset ................. 51 


\section{CHAPTER 1}

\section{INTRODUCTION}

\subsection{BACKGROUND}

Assessment of pain is an important step towards the treatment of pain. There is a need for manageable, valid and reliable tools to assess pain [10]. Accurate assessment of pain is critical for the identification of appropriate interventions and for evaluating the effectiveness of such interventions in the clinical setting [11]. Regular pain assessment is necessary for the presence of pain and for improvement, deterioration, or complications attributable to pain treatment. [11]

The use of mobile and wireless technologies such as smartphones is becoming very popular in the healthcare area, popularly known as mHealth. Lower cost and pervasive use of mobile devices are the main reasons that make healthcare faster, better and affordable. Recent research shows that mHealth has, and will continue to have a remarkable impact on the delivery of healthcare services. By paying close attention to participant's self- reported pain along with data mining based pain level detection, the healthcare industry and researchers may deliver accurate health services in pain treatment. There are two types of users in the mHealth system. One is the health care provider and the other is the patient. Also in research studies, this mHealth system can be used by the researcher and participant. Primary data collection can be increasingly convoluted due to incurring substantial costs, both money and money critical resources for researchers in particular studies [20]. 
Pain expression recognition could be useful in many applications such as detecting pain in hospital or home based patients who cannot move, speak, or remember the pain they experienced [23]. Both researcher and participant may encounter numerous challenges during the research study due to the inaccurate pain level detection and design issues of the system. Developing a smartphone-based pain study platform, which will offer seamless services to pain researcher and participant, is a challenging task.

\subsection{OBJECTIVES}

The objectives of this thesis are the followings:

- Generic pain study platform for pain research

- $\quad$ Provide researchers a device independent tool

- $\quad$ Provide participants a collaboration platform

- $\quad$ Easy collaboration between primary and secondary researchers

- $\quad$ Provide pain researchers a real-time accessible data collection tool

\subsection{THESIS ORGANIZATION}

The rest of this thesis is organized as follows:

$\square$ Chapter 2 investigates existing works in the pain research field.

$\square$ Chapter 3 analyzes the required modules of the pain study platform.

$\square$ Chapter 4 describes pain detection tool techniques and its personalization. 
$\square$ Chapter 5 discusses the key design considerations of the platform development.

$\square$ Chapter 6 presents the details of the developed platform.

$\square$ Chapter 7 analyzes the evaluation techniques and results of the platform and the pain tool.

$\square$ Chapter 8 concludes the thesis with a summary and ideas for the future works. 


\section{CHAPTER 2}

\section{RELATED STUDY}

Lack of representative data is one of the major impediments to develop a fully functional automatic pain detection tool [22]. Though several public datasets for different targeted pain study [22] are made available through various research publications but no shared platform was found for sharing pain study data during priori studies.

Facial expressions are considered a reliable indicator in pain assessment such as neonatal pain assessment etc. Here facial images are classified into four classes of facial expressions: calm, crying, moderate pain, severe pain. The classification accuracy is up to $85.50 \%$ [1]

Author employed Recurrent Neural Networks (RNNs) to automatically estimate Prkachin and Solomon Pain Intensity (PSPI) levels from face images. Personalization of the model is performed using a newly introduced facial expressiveness score, unique for each person. [2]

Three important factors were figured out to exploit in automatic pain detection: spatial information available regarding to pain in each of the facial video frames, temporal axis information regarding to pain expression pattern in a subject video sequence, and variation of face resolution. [3]

However, the ability to train deep CNNs for pain assessment is limited by small datasets with labels of patient-reported pain intensities [4], i.e., annotated datasets such as EmoPain [6], Shoulder-Pain [5], BioVid Heat Pain [7] 
Image based approaches are sensitive to noise and illumination. Local binary pattern (LBP) is more immune to these. LBP is a local texture operator to describe the image texture by comparing the gray value of a pixel $\mathrm{x}$ with the gray values of its neighbors within a predefined window of radius $\mathrm{R}$. [8]

The shape and appearance of facial features get deformed due to pain. The deformation of appearance features is extracted using local binary pattern features. [9]

\subsection{Pain Assessment Tools}

Related research study revealed 28 pain assessment tools and/or techniques. Scoring methods and rating of period varied much in terms of scale and technique from each other. Most of the tools (24 out of 28) are dependent on observations of healthcare professionals. 


\begin{tabular}{|c|c|c|}
\hline Abbey pain scale & ADD Protocol & Behavior checklist \\
\hline CNPI & Comfort checklist & CPAT \\
\hline Doloplus-2 & DS-DAT & ECPA \\
\hline ECS & EPCA-2 & FACS \\
\hline FLACC & Mahoney pain scale & MOBID \\
\hline NOPPAIN & PAINAD & PACSLAC \\
\hline PADE & PAINE & PATCOA \\
\hline PBM & PPI & PPQ \\
\hline $\begin{array}{l}\text { Observational pain behavior } \\
\text { tool }\end{array}$ & RaPID & REPOS \\
\hline Pain assessment & r use with cognitive & red adults \\
\hline
\end{tabular}

Table 1: Existing Pain Assessment Tools.

Pain tools which tried to determine pain level based on numeric values, ranges varied from 0-60. 


\begin{tabular}{|c|c|}
\hline CNPI & $0-6$ \\
\hline PATCOA & $0-10$ \\
\hline FLACC & $0-10$ \\
\hline MOBID & $0-10$ \\
\hline PAINAD & $0-10$ \\
\hline ECS & $0-14$ \\
\hline Observational Pain Behavior Tool & $0-25$ \\
\hline DS-DAT & $0-27$ \\
\hline Doloplus & $0-30$ \\
\hline ECPA & $0-44$ \\
\hline RAPID & $0-54$ \\
\hline PACSLAC & $0-60$ \\
\hline
\end{tabular}

Table 2: Numeric Score Based Pain Scale Systems. 


\section{CHAPTER 3}

\section{PLATFORM REQUIREMENT ANALYSIS}

From various previous and ongoing researches following key requirements are identified. Those are discussed below-

\subsection{MOBILE APPLICATION}

For a facial image-based pain tracking tool, the first and foremost requirement is the ability to capture facial images when patient is in pain. Taking facial images without any difficulty and transmitting it for processing without any delay is an absolute requirement. The share of Americans that own smartphones has increased to $77 \%$ from just $35 \%$ in 2011 [12]. Considering the penetration of smartphone users, this is the best available tool for a patient or patient attendee to capture a facial image via self-portrait photograph or by other means such as selfie stick etc. while the patient is feeling pain. The application for taking and transmitting this image for further processing needs to be as simple as possible without any complex usage procedure. 


\subsection{SERVER APPLICATION}

Image processing applications usually drain more power than other application due to processing of pixel by pixel of the image along with resource hungry mathematical computation [13]. This battery drain can be avoided if the heavy processing is done in server side and the result is sent back to the smartphone via internet communication [14]. The pain detection module and pain tracking system itself need to be independent from any smartphone platform. It needs to be loosely coupled and open for any foreseeable future extension without requiring any modification to the mobile application.

\subsection{USER ENROLLMENT}

Proper enrollment of users is important for identification of collected data from participants and keeping track of their association with corresponding researchers. During enrollment, generally a various degree of personal information of the participant are being collected. Privacy and security of personal data are discussed later in this chapter. One of the key requirements of the system is the ability to separate participant's personal data from the other participant's or other research studies. Another challenge in many pain research studies is finding enough relevant participants for research study. Having a system on which participants can join different research studies would alleviate the major challenge of pain study. 


\subsubsection{RESEARCHERS}

Researcher is one of the two user access level in the system. They will mainly have the administrative control of the system. They would be able to enroll participants in their research study and would be able to access only their participants pain data.

\subsubsection{PARTICIPANTS}

Participants are the patients who are willing to share their facial images along with their valuated pain data, in a scale, with research studies. They should be able to choose share data with no research study as well.

\subsection{PATIENT HISTORY MANAGEMENT}

This pain tracking tool should be able to manage the data submission history by the participants. Participants would be able to see their submission history also along with their own determined value of pain scale and the system predicted pain 
scale. Participants could also decide to join any research study with their previously provided data.

\subsection{RESEARCH APPLICATION}

A system which is built from a design that has not taken into enough consideration the elements of evolution and extensibility, may well become subject to the phenomenon known as "code rot" or "design rot" and may because of this ultimately be abandoned as the system becomes too hard to maintain and extend. [16] The applications should be customizable for an end user, while allowing the underlying application to be modernized without losing any user customizations [15]. Meanwhile achieving openness of the application it should also be ensured that the application is closed for any modification from outside. According to Interfacesegregation principle (ISP), no component should be required to rely on methods it does not use [17]. 


\section{CHAPTER 4}

\section{PAIN DETECTION AND PERSONALIZATION}

\subsection{COMPONENTS}

\section{Visual Analog Scale (VAS):}

The visual analog scale (VAS) is a psychometric response scale which can be used to assess the amount of pain that a patient feels ranges across a continuous scale from none to an extreme amount of pain [24]. It requires a very little training and the pain VAS has been found acceptable to patients [25] [26] [27].

\section{YCbCr Colorspace:}

$\mathrm{YCbCr}$ is one of the widely used color space in image processing area. It is a scaled and offset version of YUV color space. In comparison to $\mathrm{RGB}, \mathrm{YCbCr}$ is luma-independent. There are 3 components in $\mathrm{YCbCr}$ color space. In $\mathrm{YCbCr}$ format, $\mathrm{Y}$ represents luminance information whereas $\mathrm{Cb}$ and $\mathrm{Cr}$ are two color-difference components. Component $\mathrm{Cb}$ and component $\mathrm{Cr}$ are the difference between blue component and a reference value and difference between red component and a reference value.

\section{Eigenface:}

An Eigenface is the projection of face images onto a feature space that ranges a significant variation among known faces. Principal components are identified by principal component analysis (PCA) which is manifested by the eigenvectors corresponding to the highest eigenvalues. 


\subsection{PAIN DETECTION ALGORITHM}

The face image was taken as input from mobile device's front camera and then color segmentation through $\mathrm{YcbCr}$ color space followed by density regularization and luminance

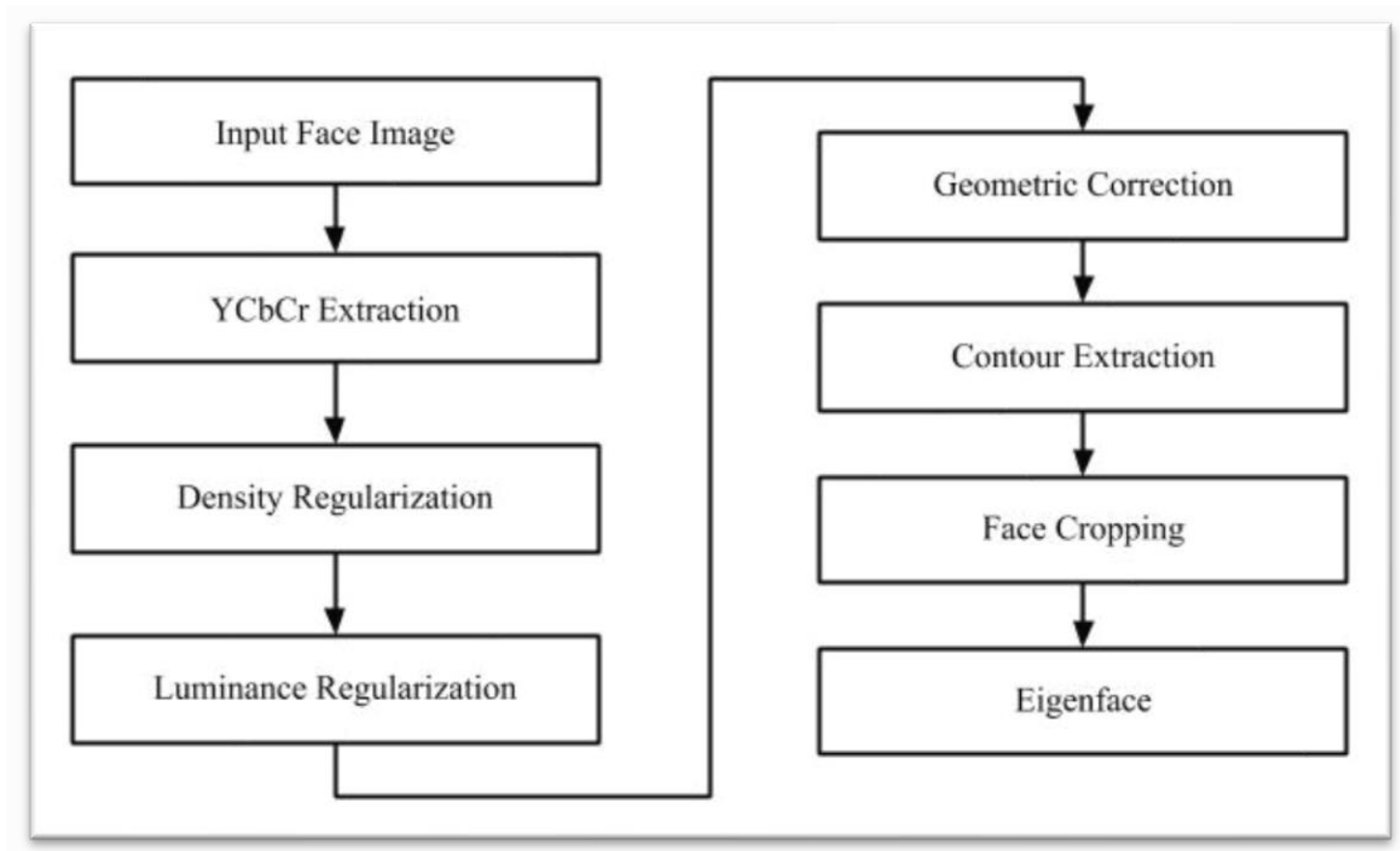

Fig 1: Face Transformation

regularization. After these, geometric correction was performed with contour extraction.

Now for a training set of $M$ images, and that each image is represented as an $i \times j$ matrix of pixels values. Now let $n=i j$.

These $M$ images are transformed into column vectors of length $n$. An arbitrary image, $I_{1}$, from the total M-sized collection is considered as follows: 


$$
I_{1}=\left[\begin{array}{cccc}
P_{11} & P_{12} & \ldots & P_{1 i} \\
\vdots & \vdots & \vdots & \vdots \\
P_{i 1} & P_{i 2} & \ldots & P_{i j}
\end{array}\right]
$$

Now $I_{l}$ is transformed into a column vector, $\Gamma 1$ by concatenating the columns of $I_{1}$ :

$$
\Gamma 1=\left[\begin{array}{c}
\left(\begin{array}{c}
P_{11} \\
P_{21} \\
P_{i 1}
\end{array}\right) \\
\left(\begin{array}{c}
P_{12} \\
P_{22} \\
P_{i 2}
\end{array}\right) \\
. . \\
\left(\begin{array}{c}
P_{1 j} \\
P_{2 j} \\
P_{i j}
\end{array}\right)
\end{array}\right]
$$

After performing this transformation on all images, $M$, following set $S$ is obtained as below:

$$
S=\left\{\begin{array}{lllll}
\Gamma_{1}, & \Gamma_{2}, & \Gamma_{3}, & . & , \Gamma_{M}
\end{array}\right\}
$$

Then the mean image $\Psi$ is computed as follows -

$$
\Psi=\frac{1}{M} \sum_{x=1}^{M} \Gamma_{x}, \text { where } x \in N \text { and } 1 \leq x \leq M
$$

Now taking the difference of each $\Gamma_{x}$, a new set of vectors is obtained which is represented by $A-$

$$
A=\left[\Phi_{1}, \Phi_{2}, \ldots, \Phi_{M}\right]_{n \times M} \text {, where } \Phi x=\Gamma x-\Psi, x \in N, 1 \leq x \leq M
$$


The covariance matrix $C$ has been calculated using the following equation -

$$
C=\frac{1}{M} \sum_{x=1}^{M} \Phi_{x} \Phi_{x}^{T}=A A^{T}
$$

Finding out eigenvectors from the covariance matrix is a huge computational task considering a typical image of size $N X N$ becomes a vector of dimension $N^{2}$ or, equivalently, a point in $N^{2}$-dimensional space. Since $M$ is far less than $N$ by $N$, a M by M matrix was constructed.

$$
L=A^{T} A
$$

$M$ Eigenvectors, $v_{l}$ of $L$ were found. These vectors $\left(v_{l}\right)$ determine linear combinations of the $M$ training set face images to form the Eigenfaces. $u_{1}$

$$
u_{1}=m \sum_{k=1}^{M} v_{x k} \Phi_{k}, \text { where } x=1,2,3, \ldots . ., M
$$

and finally, Eigenface was obtained. The dimension of the weight vector corresponds to the training set of facial images. To detect the pain level of a test image, the test image is projected onto the Eigenspace of the training images. By using linear combinations of these Eigenfaces, each image can be reconstructed with a varying degree of resemblance. This projection provides the coefficients of our linear combination for each image, as well as the coordinates of each face within the Eigenspace. As the calculated eigenvectors are unit vectors, the projection of any face image $f$ onto an Eigenface $E_{i}$ is simply the dot product of $f$ and $E_{i}$, or $f \cdot E_{i}$ and thus angular distance is measured according to the following equation - 


$$
d(A, B)=\frac{A \cdot B}{|A| \cdot|B|}
$$

\subsection{PERSONALIZED CONTINUOUS LEARNING}

Like any other machine learning problem, scope of facial expression learning can be broadly categorized to two categories, shown Fig 2, - 1) Generic Learning (Cross-sectional Learning) and 2) Personalized Learning (Longitudinal Learning).

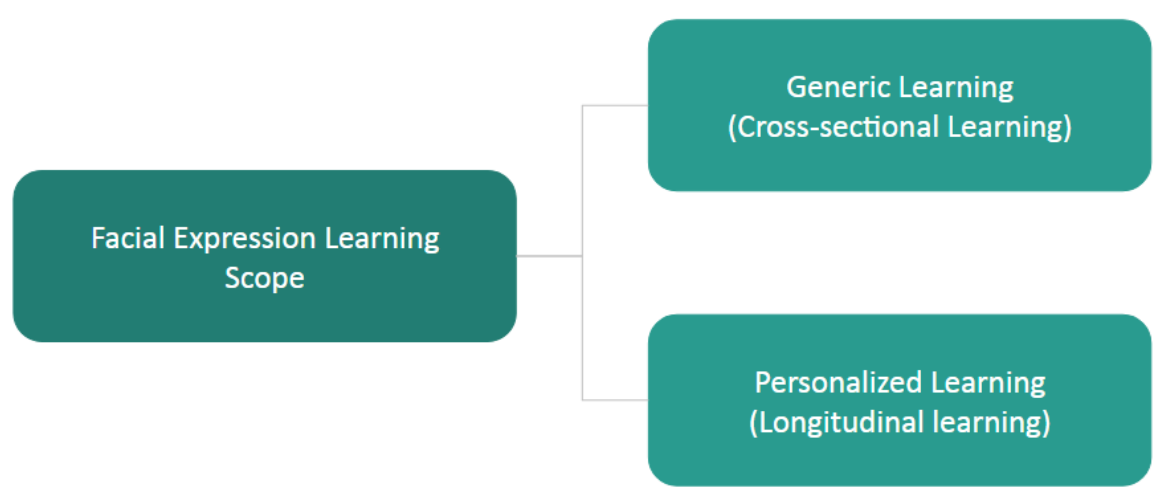

Fig 2: Facial Expression Learning Scope

In the cross-sectional learning model, model is trained by the facial images of the whole population and tested with the facial images of the test subjects. With respect to cross-sectional learning, model is trained only the by facial images of the test subject and tested with the images of corresponding subjects only. 
Again, based on learning period, facial expression learning can be classified into two different approaches, shown Fig 3, - 1) One-time learning and 2) Continuous learning.

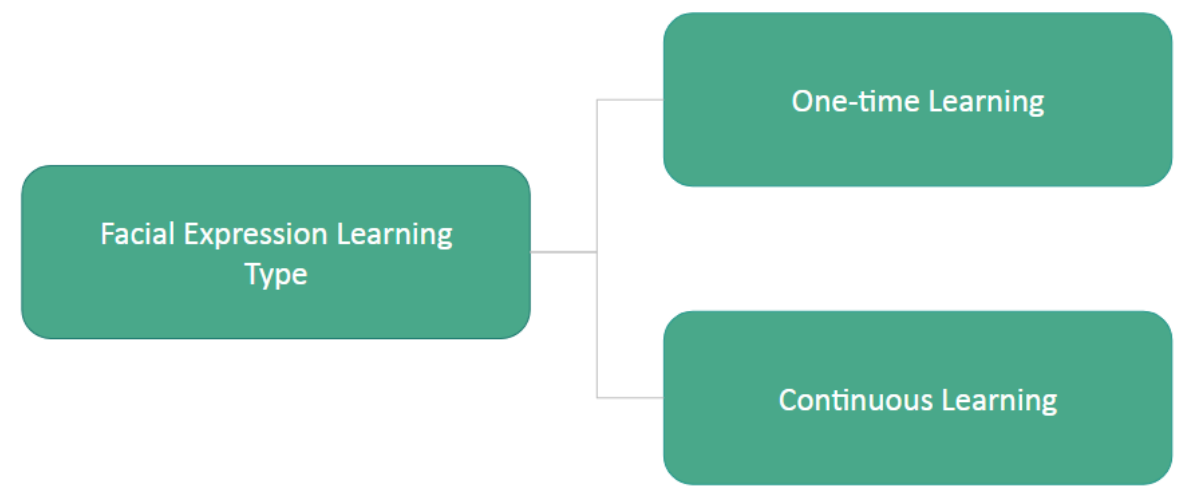

Fig 3: Facial Expression Learning Period

One time learning aka traditional learning model is based on learn once-test multiple model. The limitation of this approach is adaptability and scalability. On the other side, continuous learning refines the prediction model and in the end offers improvement in the offered service. Continuous learning generally performs better in scenarios where the data distribution is generally same but new data keeps coming over the time.

Expression of faces due to pain differs greatly from person to person depending on demographics such as age, gender etc. To circumvent this problem a personalized pain detection tool based on continuous learning is the solution for an evidence based pain tracking tool. 


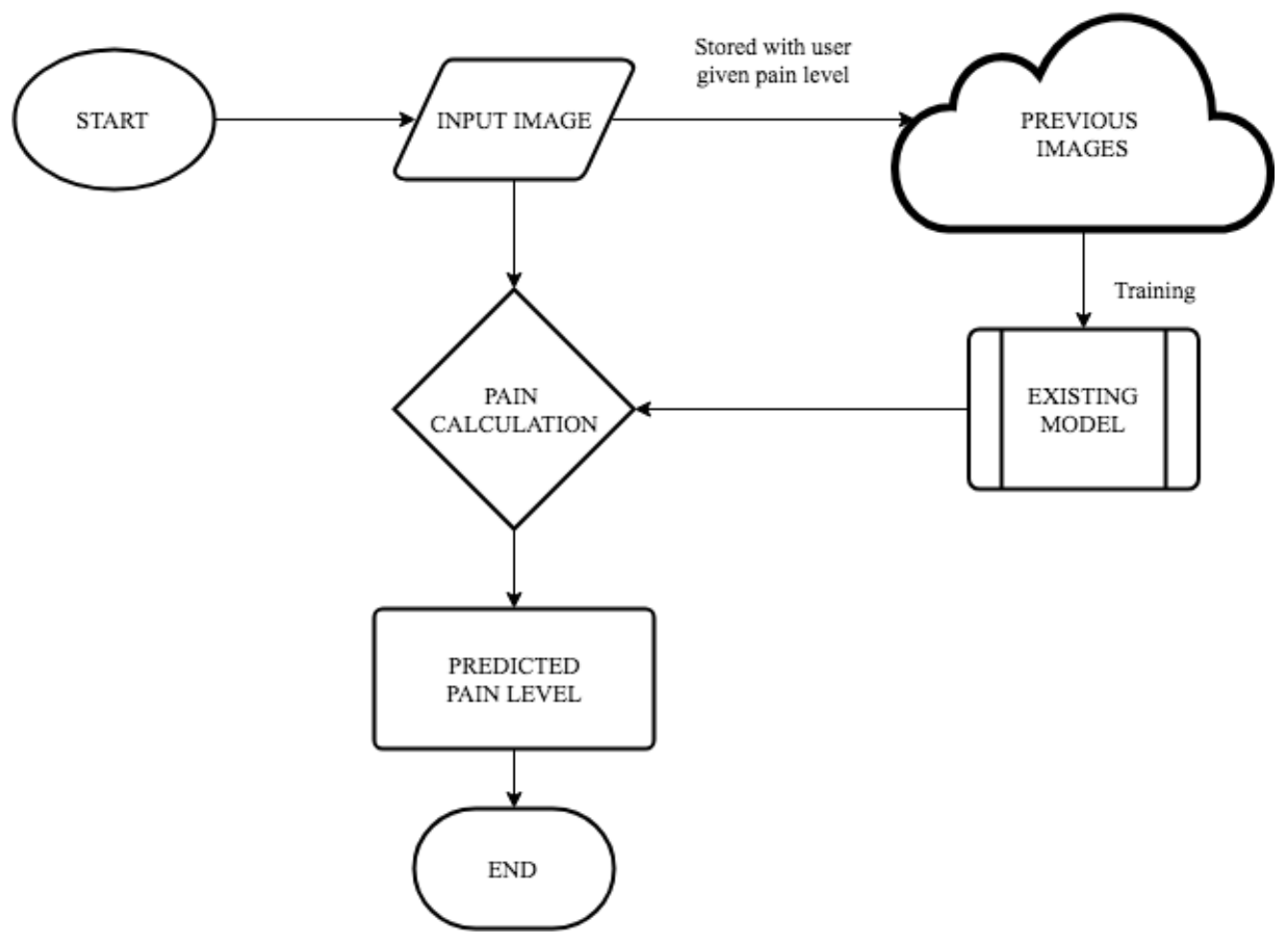

Fig 4: Personalized Training

The same pain detection algorithm described in the above section is used to device a personalized trained model. An existing model is prepared and stored beforehand using previously collected images for each user. Then the user input face image is tested to predict current pain level. The predicted pain level is displayed to the user and a new training model is prepared using this current input image combining with previously stored images. This new training model replaces the previous training model for this particular user and doesn't affect other users pain recognition process. The lifecycle of the tools can be represented by Fig 5 . 


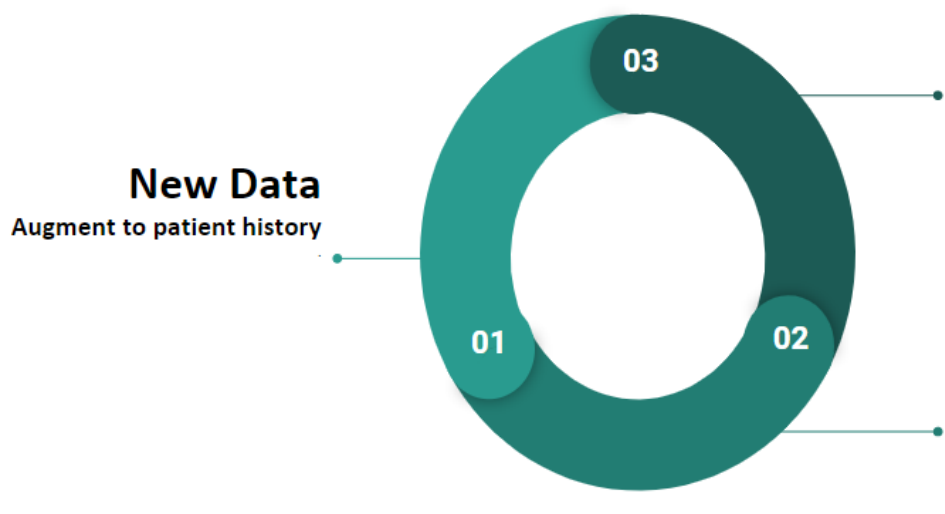

Prediction

Predict the pain level using latest machine learning model

\section{Re-train}

Augment the new data with previous training data to update model

Fig 5: Continuous Learning Lifecycle 


\section{CHAPTER 5}

\section{SYSTEM DESIGN AND SYSTEM DESCRIPTION}

A good software design can assure the development of successful software. Following sections discuss a few good practices of designing software [18].

\subsection{DEVELOPMENT PROCESS AND REQUIREMENTS ANALYSIS}

The objective of choosing an appropriate development lifecycle process for software development is to deliver a quality product for business success and to ensure best utilization of resources. Based on the functional requirements and delivery requirements it is vital to select a process which will ensure timely delivery ensuring desired level of quality. The regularly used methodologies enclose direction about how to execute the development process.

There are currently quite a few popular software development lifecycles. One of the popular methods is Agile. Agile is a variant of lifecycle where deliverables are submitted in stages [19]. But above all, it is more important to execute the chosen lifecycle properly then choosing a better one but not executing it.

Requirement analysis includes compiling, analyzing and agreeing on requirements. It is absolute essential for a successful project. It is not necessary to have all the requirements locked before any system architecture or system design or development is done. But a clear understanding of overall requirements is essential before designing a system. 


\subsection{ARCHITECTURE AND DATABASE DESIGN}

The platform architecture represents a software infrastructure that satisfies the business and service-level requirements of a system. Selection of software architecture is very vital to ensure the extensibility and adaptability - two critical cornerstones of any research application. Careful consideration of users, the system and the research goals is required during system design to avoid failure of identifying key scenarios, common problems or long term consequences.

For any challenging research focused application like pain study platform, tradeoffs are likely and a balance is must often be found between research agenda and availability of critical of resources such as time and money. There are quite a few recognized architectural patterns and styles in the software industry, listed in Table 3, based on functional purposes, communication philosophy, and modular dependency etc.-

\begin{tabular}{|c|c|}
\hline $\begin{array}{c}\text { Client-Server (2-tier, 3-tier, cloud } \\
\text { computing) }\end{array}$ & Component-based \\
\hline Event-driven & Multi-layered \\
\hline Service-oriented & Microservices \\
\hline Peer-to-peer & Monolithic application \\
\hline Shared-nothing architecture & Rule-based System \\
\hline
\end{tabular}

Table 3: Common Platform Architectures and Styles. 
Being a data-focused research application, collected data from patients is the asset of this platform. A thoroughly designed database is essential to paramount the goal while developing any research focused platform. The activities that focus on the design of the database structure that will be used to store and manage end-user data are referred by database design. According to Carlos and Steven in their book [30] "Even a good DBMS will perform poorly with a badly designed database".

Having an Entity-Relationship model (or ER model) is essential to establish the logical relationships of entities in order to have a planned database design. Designing and developing a platform without ER diagrams are not unheard of, though it creates opportunities for misunderstanding the requirements during the development process.

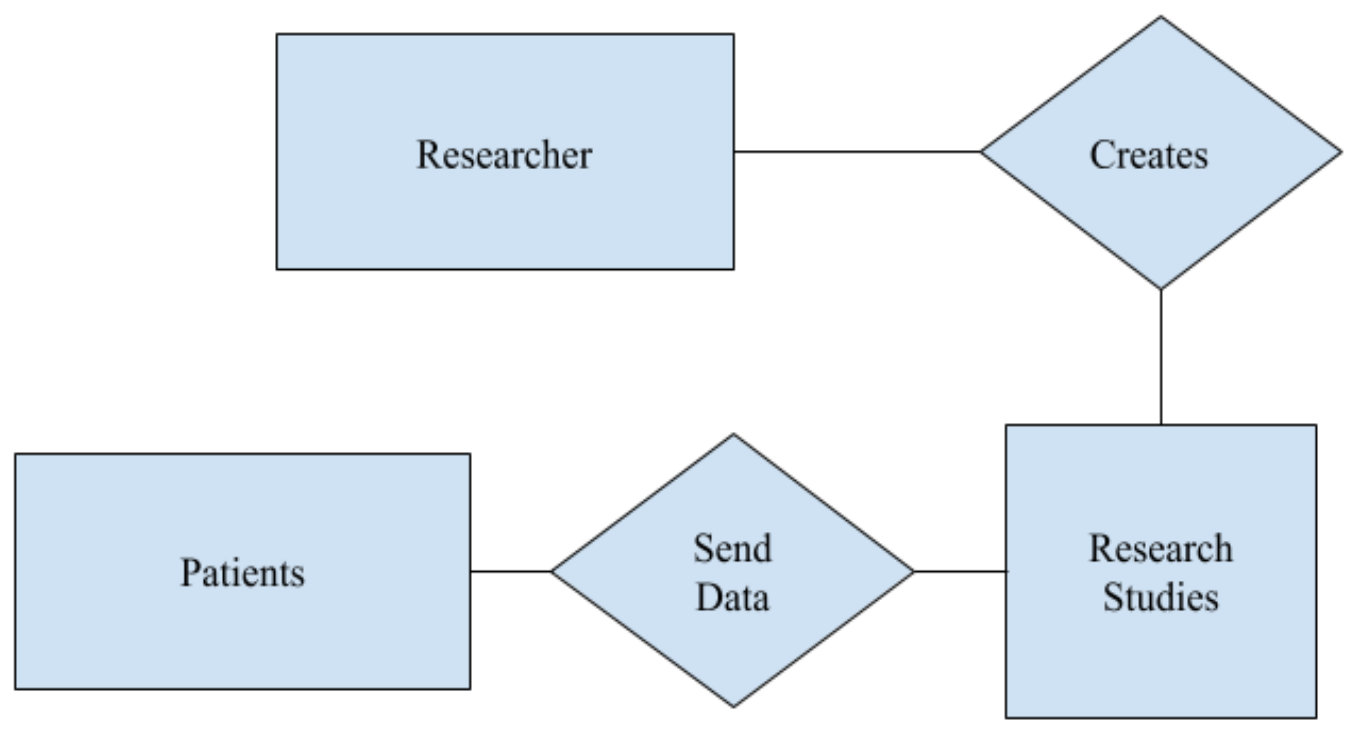

Fig 6: Example of ER Diagram 
An example is given in Fig 6. The elements researcher and patient are described using ER diagrams. The rectangular elements represent the entities while the diamond items denote the relationship between entities.

\subsection{USABILITY}

As stated in an article by Microsoft in [32], software usability represents an approach that puts the user, instead of the system, at the center of the process where this user-centered approach embraces users concerns and dictates that the comforts of users should be foremost in any design decisions. Usability of a mHealth system is discussed in this chapter.

\subsubsection{AILING USER'S POINT OF VIEW}

As mHealth tools are primarily targeted for ailing users. Previous studies show a simple and straightforward software user interface of mHealth tool is essential to enhance user experience among ailing users [33]. Even user interface components e.g. the size of text and buttons etc. also affects the usability of mHealth tools for ailing users with dexterity impairments [34].

The following characteristics have been identified from literature -

Acceptance - Patient perception is of paramount when it comes to ensuring success of a tool and the patient's point-of-view must be considered throughout the development and design process [35]. 
Convenience - The tool must be convenient, unobtrusive for the user to wear [36], easy to learn and undemanding to use [37].

\subsubsection{RESEARCHER'S POINT OF VIEW}

Healthcare practitioners, whether researchers, physicians or other, need accurate and reliable information from the system in order to make effective interventions for patients and for better research outcomes. They are mainly focused on -

Acceptance - According to a study on a Mobile Clinical Decision Support (mCDS) system conducted at a UK Teaching hospital [35], the perception of the healthcare practitioner is affected by various factors, including perceived usability, perceived benefits of using, perception other people has, and self-motivation.

Quality of service - From a practitioner's perspective, the targeted use should be easily achievable, and the system should be able to provide reliable data [38]

\subsection{SYSTEM SECURITY AND PRIVACY}

It is fundamental to consider system security and user's personal data privacy for any software systems especially for mHealth systems which deal with sensitive health information. But it is very challenging to strike out a balance between usability and privacy [39]. There are systems which could not achieve high usability due to 
emphasizing more on privacy, while systems focused on usability are not highly secure [40].

\subsubsection{ROLE-BASED ACCESS}

Role-based-access-control (RBAC) is a security approach to regulate system access to authorized users [41] [42]. This access control system is evidently popular choice for enhanced security in many enterprise systems directly or indirectly [43]. In RBAC access to resources are granted based on roles and roles are generally defined by authority and responsibility for resources.

\subsubsection{APP AUTHENTICATION}

App authentication refers to the authentication and identification of permitted users through mobile applications. A poorly designed registration screen or sign-in mechanism may lead to poor user engagement [46]. Mobile app users typically lack motivation in filling out forms and creating accounts especially for newer platforms. To ensure more participation in a mobile based mHealth research system, it is necessary to develop a simplified authentication process in the mobile apps.

\subsubsection{HIPAA COMPLIANCE}

As described earlier, security and privacy are most two imperative design issues during planning of a research focused digital healthcare application. Whereas 
the Protected Health Information (PHI) is dealt through HIPAA (Health Insurance Portability and Accountability Act) Privacy Rule, the electronic Protected Health Information (ePHI) is dealt by HIPAA Security Rule.

Any health-related data which are generated, collected, retained or transmitted electronically needs to be safeguarded by the application and it must adhere to HIPAA Security Rule [44]. According to U.S. Department of Health \& Human Services, "The Security Rule requires appropriate administrative, physical and technical safeguards to ensure the confidentiality, integrity, and security of electronic protected health information". Any data transaction of the application with a user e.g. participant or researcher, any data communication between internally independent modules either hosted in the same or different servers and the online system itself should be secure and encrypted when it comes to the human subject. In a pain research study, a participant may need to share various types of personal information with researchers. Some secure protocols like HTTPS (Hypertext Transport Protocol Secure) can ensure security in data communication medium over internet. The use of HTTPS helps to prevent eavesdropping and man-in-the-middle attacks. With respect to HTTP, HTTPS generally uses one of two secure protocols to encrypt communications - TLS (Transport Layer Security) or SSL (Secure Sockets Layer). Using host servers which are already HIPAA compliant is also another way of achieving security. Example of private data includes participant's name, address, phone number etc. Participant's health related data is also private data. User's credentials such as username, passwords, passcodes or answers to security questions are also private information. All of this information should be encrypted during storing into the database or transmitting through medium like internet. 
Medicine and health related study should adhere to HIPAA Privacy Rule [45]. According to U.S. Department of Health \& Human Services, "The Rule requires appropriate safeguards to protect the privacy of personal health information, and sets limits and conditions on the uses and disclosures that may be made of such information without patient authorization. The Rule also gives the patient's rights over their health information, including rights to examine and obtain a copy of their health records and to request corrections". 


\section{CHAPTER 6}

\section{PLATFORM IMPLEMENTATION}

Features and requirements of the developed platform are described in this chapter.

\subsection{PLATFORM ARCHITECTURE AND COMPONENTS}

As described in previous chapters, one of the primary goal of this pain study platform is to enabling researchers and willing participants to share their pain data with each other. Another primary goal is develop an evidence based continuous learning tool. The development of the tool is a very a challenging research problem and the decision of using any particular algorithm affects the research results. To ensure adaptability for any future decisions to use different algorithm for the tool or to allow deployment of different algorithms for differently targeted pain study it is imperative that the platform itself needs to very loosely coupled with the pain tool.

After careful consideration of the future prospects, to enable support for a range of techniques and devices_-spanning web, mobile, Internet of Things, and wearable of the platform, it was clear that microservices architecture suits better this type of research focused mHealth systems.

Inherently, microservice architecture is a method of developing software applications as a suite of small, independently deployable, modular services. Each service is responsible to serve a business goal and runs a unique process and communicates through a well-defined, lightweight mechanism. Microservices architecture allows scaling specific functions of the desired components, without affecting the entire 
application which makes the whole application maintainable and versatile for future extensibility.

Following the microservice architecture, our platform is decomposed to couple of following microservices -

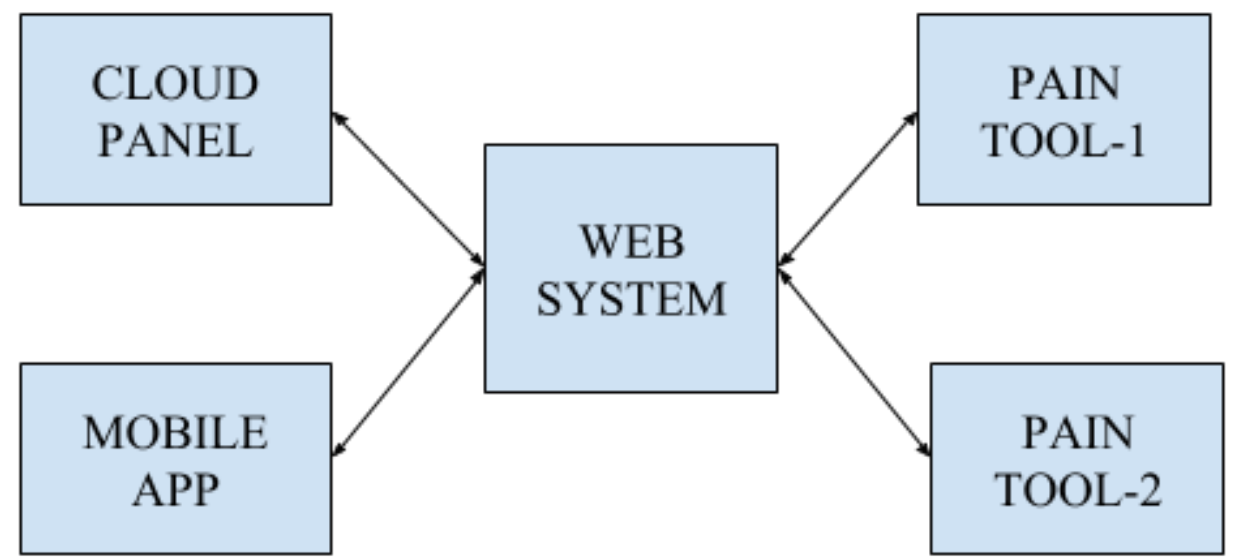

Fig 7: Implemented Microservices Architecture.

Currently there are four (4) microservices developed in the platform -

1) Cloud Panel: Cloud panel is intended to serve the researchers to conduct researches and enroll participants for the research program.

2) Mobile App: Mobile app is intended to serve the willing participants to share the pain data with the platform.

3) Web System: Web system is responsible to serve the cloud panel and the mobile app by offering storage of generated data and utilize the designated pain-tool microservice to determine the predicted pain level.

4) Pain Tool: Pain-tool is responsible for predicting the pain level by using a trained model and then retraining upon availability of new training data. 


\subsection{SYSTEM COMMUNICATION}

Theoretically microservices can communicate with each other either using asynchronous protocols such as The Advanced Message Queuing Protocol (AMQP) or synchronous protocols such as HTTP/REST. But in most cases, REST (Representational State Transfer) is a useful integration method because of its simplicity over other protocols.

Each microservice communicates with each other by using well-defined REST-ful Application Programming Interfaces (API). An API gateway was developed which acts as the entry point for the clients of the micro-services. Clients don't call services directly rather the API gateway is called, which forwards the call to the corresponding services on the backend.

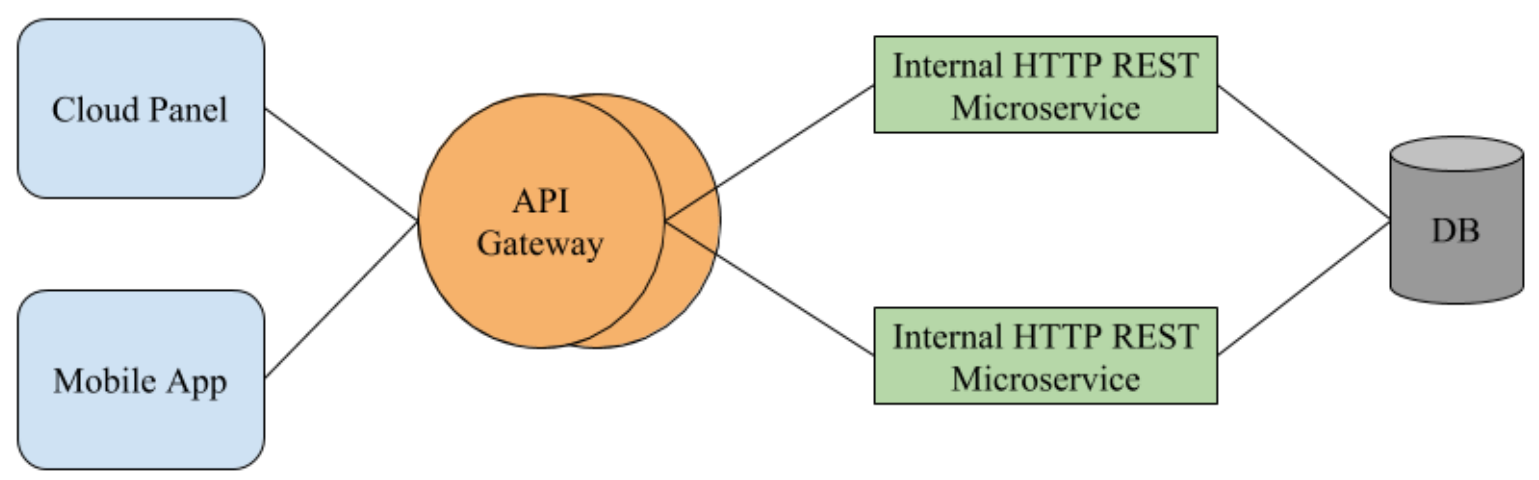

Fig 8: Microservice Communication Protocol. 


\subsection{TOOLS USED}

Different types of tools were used during development of our platform. We have highlighted major of them in this section. Those are-

- PHP

PHP (Hypertext Preprocessor) is a widely used open source server-side scripting language. . Though PHP is a general-purpose scripting language but it is especially suited to web development. PHP code can be embedded into HTML (Hypertext Markup Language) code. It can also be used in combination with different kind of web template systems, web content management systems and web frameworks [47]. In our platform, we have used PHP 5.6 in a web framework.

- Yii Framework 2.0

Yii (pronounced as Yee) is a component-based, high-performance PHP framework [48]. It's component-based architecture and sophisticated caching support facilitates developers to build modern web application quickly. There are also quite a few good PHP frameworks like Laravel, Symfony, Zend etc. To develop our pain study platform, we have used Yii as it is a stable and component based framework, well-supported by widespread open source community which allows extending existing functionalities with less resources. Project structure in Yii follows Model View Controller (MVC) pattern. As a full-stack framework, Yii provides many proven and ready-to-use features such as ease of creating forms with basic CRUD 
operations, asynchronous form validation using AJAX, various common preventive measures for better security of the web application. Adding new components, dependency libraries or maintaining previously used components based on specific versioning by Composer, an application-level package manager is very easy in Yii framework . Availability of detailed and well-maintained documentation and great developer community enables Yii a strong framework choice for most kind of web application development.

- $\quad$ Bootstrap 3.3.7

Bootstrap is free, open-source and one of the most popular front-end design library. It contains HTML- and CSS-based design templates for designing responsive web application. According to one of the founder creator of Bootstrap, Twitter developer Mark Otto: "A super small group of developers and I got together to design and build a new internal tool and saw an opportunity to do something more. Through that process, we saw ourselves build something much more substantial than another internal tool. Months later, we ended up with an early version of Bootstrap as a way to document and share common design patterns and assets within the company." [48]. It is compatible with all major browsers such as the latest versions of the Google Chrome, Firefox, Internet Explorer, Opera and Safari browsers. In our developed pain platform, Bootstrap is used to achieve responsive web design for the cloud panel so that the researchers can use the panel from any screen size devices such as desktop computers, laptops, smartphones, tablets etc. 
- MySQL

MySQL is a very powerful and open-source Relational Database Management System (RDBMS). With highly performing ACID compliant transactional capabilities and scalability, MySQL has become one of the most popular database choices for web application development. Between two editions - the open-source Proprietary Enterprise Server and the MySQL Community Server, Community Server was used in our pain study platform.

- MATLAB and MATLAB Compiler Runtime (MCR)

MATLAB (matrix laboratory) is a proprietary high-level language and interactive programming environment for numerical computing and visualization developed by MathWorks Inc. It provides an environment for researchers and programmers to develop algorithms and to analyze and visualize data. The MATLAB Compiler Runtime (MCR) is a standalone set of shared libraries that runs compiled MATLAB applications or components without installing MATLAB on computers.

\subsection{SERVER CONFIGURATION}

Cloud Panel and the Pain Tool - Two (2) of the four (4) microservices of our platform are hosted in two (2) different web servers.

Cloud panel is hosted in Apache HTTP Server using a LAMP stack. Apache is one of the most popular web servers for hosting web applications. It is capable of 
running in a process-based, hybrid or even-hybrid mode. It is scalable and flexible with different kind of environments while providing credible security measures.

The pain tool is hosted in a Tomcat server, an open-source java application server. Tomcat is a significantly lightweight web server in comparison to other available java application servers such JBoss, Glassfish etc. but it offers modularity which is very much essential for research applications to incorporate future inclusion of newer components.

\subsection{SYSTEM DESCRIPTION}

The purpose of our pain study platform is to serve researchers in research collaboration and willing pain patient participants to share their pain data with multiple researches simultaneously. Our platform can be divided into two major interfaces: researcher and patient. 


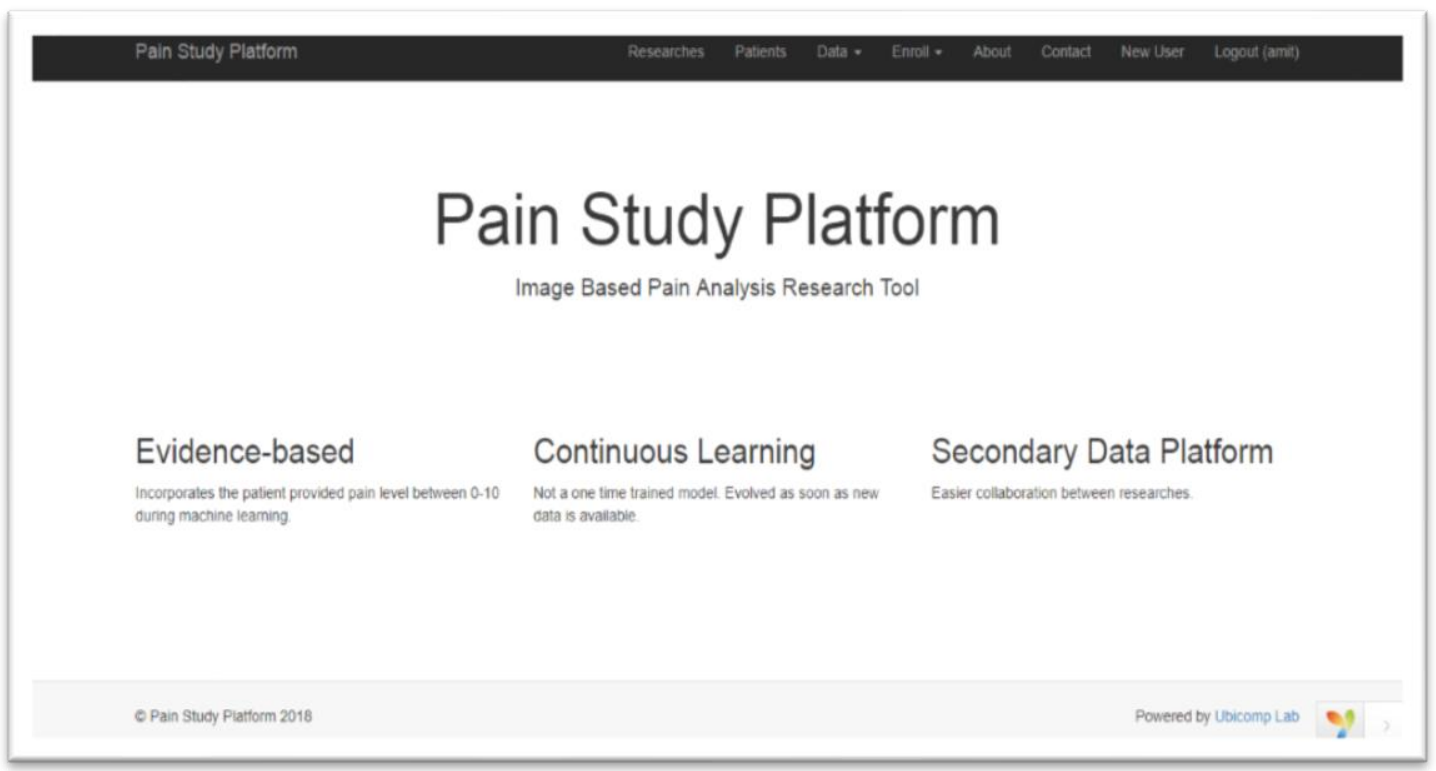

Fig 9: Pain study platform.

At the beginning Admin users of the platform would need to create new Research in the panel. Any researcher or patient is essentially an User also. An admin can enroll an user as researcher for an previously created research. Multiple researchers can be enrolled in a single research. Again, an individual researcher can be enrolled into one or more than researches.

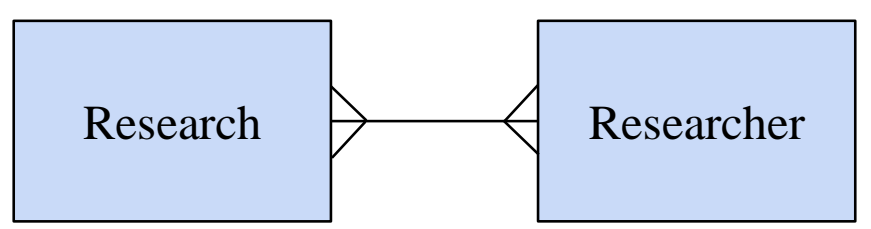

Fig 10: Research-Researcher Relationship.

On the other side, patient is also a user in the system. In general, this kind of research focused platforms generally requires more control over user sign up simultaneously simplified user enrollment is also paramount in order to encourage participation. So 
with oversight and simplicity in mind, participants were required to be created by admin user and a one-time passcode was created in the system for the user.

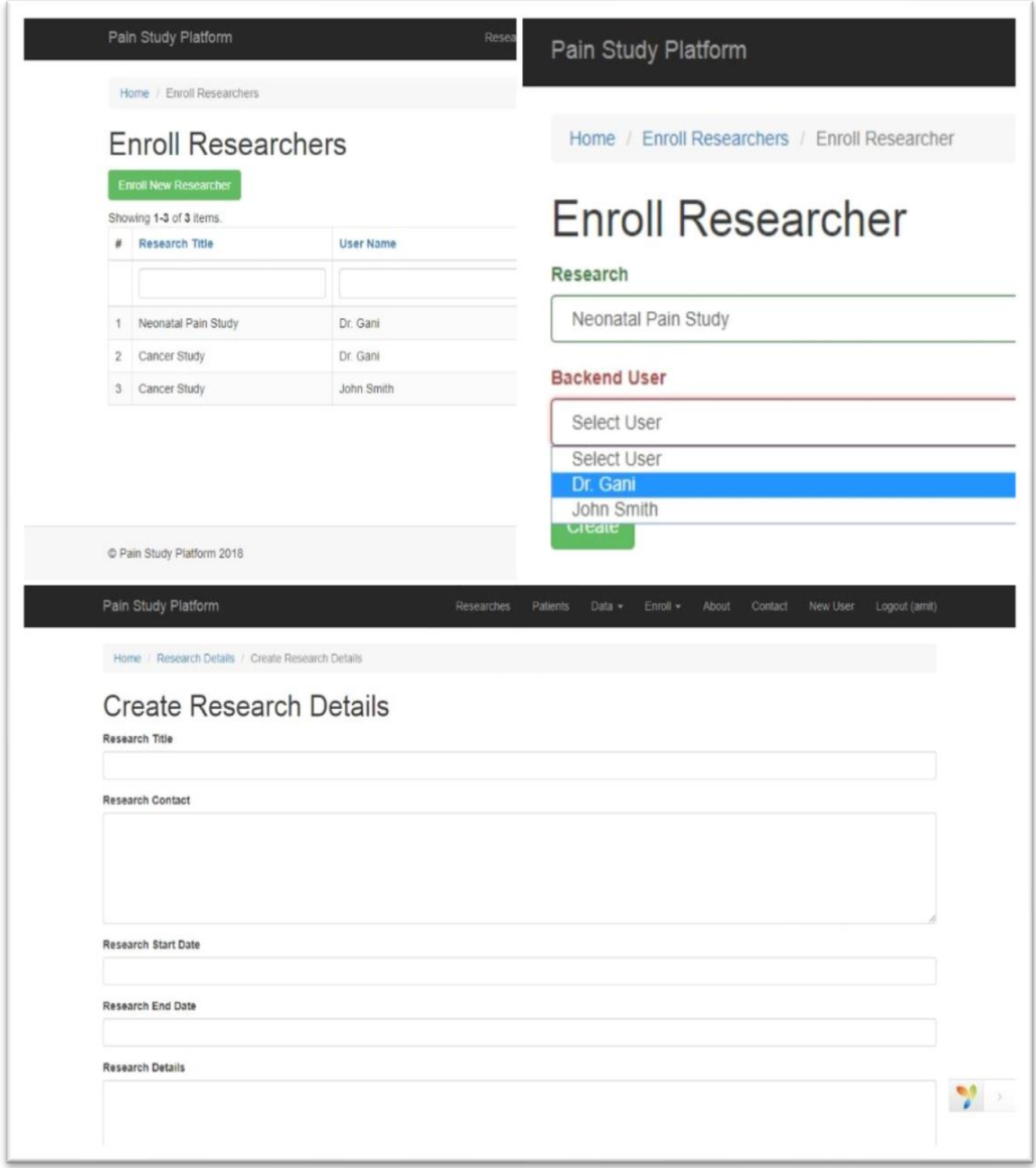

Fig 11: Research-Researcher Module Screenshots. 
That passcode was to be provided by the research program to the user and user would use that one-time passcode in the app during the first-time app launch.

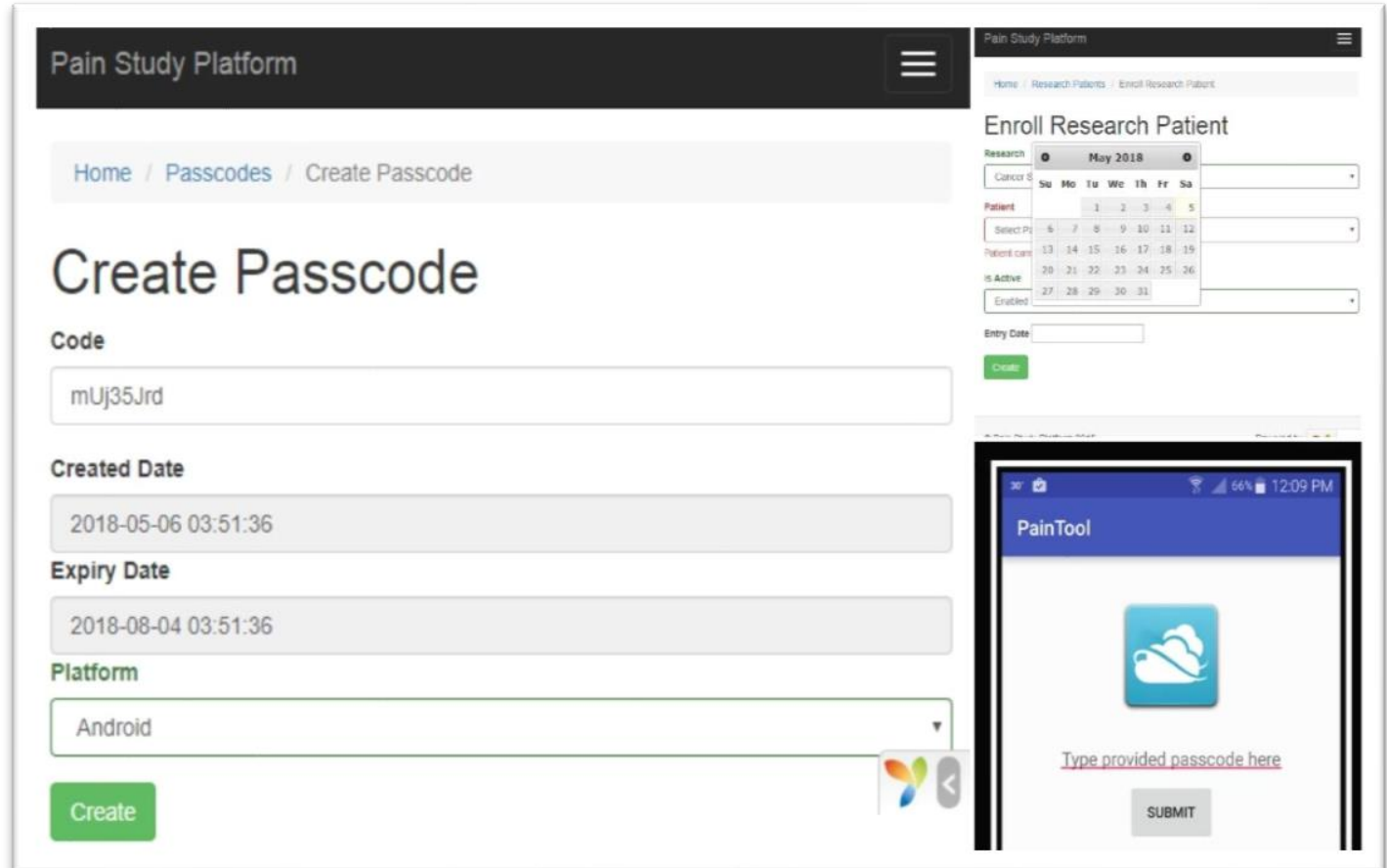

Fig 12: Patient Enrollment Module Screenshots.

To share their pain data, a patient would require to take an photo of his/her face by front camera of the app and submit the facial image along with patients own determined pain value in the VAS scale between 0-10 where 0 (zero) means no pain at all and 10 (ten) means highest pain the patient had ever experienced. Each submission of pain data along with the pain tool predicted pain value is stored in the system to be shared with the enrolled researches. Patients are also able to see their full pain data submission history in the app. 


\section{CHAPTER 7}

\section{EVALUATION}

This chapter discusses the evaluation methodologies on different test subjects and describes the results.

\subsection{PLATFORM EVALUATION}

Evaluation of a software artifact is a challenging task and requires incorporating both subjective and objective evaluation. But Researchers (Nabil et al., 2011) have found that a software artifact can be assured and measured when it is breakdown into measurable constructs [50]. According to the ISO standard on quality models (ISO/IEC 25010:2011), the main purpose of software quality evaluation is to provide quantitative reference for software products evaluation. The ISO/IEC 25010:2011 quality factors as shown in Table 4 are functional suitability, reliability, usability, performance efficiency, maintainability, portability, security and compatibility [51] [52]. 


\begin{tabular}{|ll|}
\hline Functional suitability & $\begin{array}{l}\text { Degree to which the existence of functions meets } \\
\text { stated or implied needs }\end{array}$ \\
Reliability & $\begin{array}{l}\text { Software capability under given conditions for a } \\
\text { specified period of time. }\end{array}$ \\
Performance Efficiency & $\begin{array}{l}\text { The relationship between the software's performance } \\
\text { level and the required amount of resources under } \\
\text { stated conditions. }\end{array}$ \\
Security & $\begin{array}{l}\text { Attributes that determine the effort needed for } \\
\text { learning and the assessment of usage by a set of } \\
\text { users in a specified context of use. }\end{array}$ \\
Compatibility & $\begin{array}{l}\text { Degree to which access to information and data are } \\
\text { protected according to the level of authorization. }\end{array}$ \\
Capability of the software system to interoperate \\
with other systems in terms of data exchange without \\
any detrimental impact on itself or other products.
\end{tabular}

Table 4: Eight quality characteristics of ISO/IEC 25010:2011.

Our developed pain study platform is evaluated in light of above mentioned ISO/IEC 25010:2011 quality factors as shown in Table 5 - 


\begin{tabular}{|c|c|}
\hline Functional suitability & $\begin{array}{l}\text { Meets functional requirements to allow collection of } \\
\text { pain data and collaboration between researches and } \\
\text { willing patient participants. }\end{array}$ \\
\hline Reliability & $\begin{array}{l}\text { All data transactions are fully ACID compliant and } \\
\text { any kind of change in data reflects immediately to } \\
\text { the researchers and participants. }\end{array}$ \\
\hline Performance Efficiency & $\begin{array}{l}\text { The required computational cost for the platform is } \\
\text { very low at best. }\end{array}$ \\
\hline Usability & $\begin{array}{l}\text { The developed pain study platform is very easy to } \\
\text { learn. It is designed with minimalistic view } \\
\text { considering the seamless engagement of the target } \\
\text { users. }\end{array}$ \\
\hline Security & $\begin{array}{l}\text { Role based access is implemented for different } \\
\text { authorization level of collected data. One time } \\
\text { passcode is also implemented for patient verification. }\end{array}$ \\
\hline Compatibility & $\begin{array}{l}\text { Implemented micro-service architecture enables the } \\
\text { platform to be compatible with other platform or } \\
\text { systems. }\end{array}$ \\
\hline Maintainability & $\begin{array}{l}\text { Platform is developed entirely with well documented } \\
\text { and widely popular open source tools and } \\
\text { programming stack. Model-View-Controller (MVC) } \\
\text { design pattern allows maintaining the code base with } \\
\text { minimum effort from developers. }\end{array}$ \\
\hline Portability & $\begin{array}{l}\text { The pain study platform is very easy to deploy and } \\
\text { the whole platform along with already collected or } \\
\text { generated data can be ported to another environment } \\
\text { with minimal cost. }\end{array}$ \\
\hline
\end{tabular}

Table 5: Platform Evaluation based on ISO/IEC 25010:2011.

\subsection{PAIN DETECTION TOOL EVALUATION}

Both cross-sectional and longitudinal studies were performed on the obtained pain dataset. Two (2) experiments were done to evaluate the pain detection tool. 


\subsubsection{DATASET}

A dataset of pain patients suffering with cancer was collected from a previously done cross-sectional and longitudinal study conducted in Bangladesh, Nepal and South Dakota in United States [10] [29]. The data collection protocol was approved at Marquette University and by the responsible ethical review boards in Bangladesh, Nepal and Rapid City, South Dakota in the United States[10][29]. The dataset has four hundreds and ten (410) longitudinal facial images of four (4) different subjects and thousands cross-sectional facial images of five hundreds and thirteen (513) subjects. Each image was labeled with patient given numerical value of pain in the range of $0-10$ where 0 means no pain and 10 means the highest level of pain.

\subsubsection{CROSS-SECTIONAL EVALUATION}

During the cross-sectional evaluation of the pain tool, the model was trained with all cross-sectional facial images of different subjects and the full longitudinal dataset of four subjects was used as test data. Predicted pain level range was also the same as input pain level 0-10 where 0 means no pain and 10 means the highest level of pain. Then the range of pain level (0-10) was transformed into three (3) categorical levels: low (0-3), mid (4-6) and high (7-10). This classification into categories is similar to the Brief Pain Inventory which has been proposed and validated across different cultures [29]. Residuals of each level were observed for four (4) subjects. 

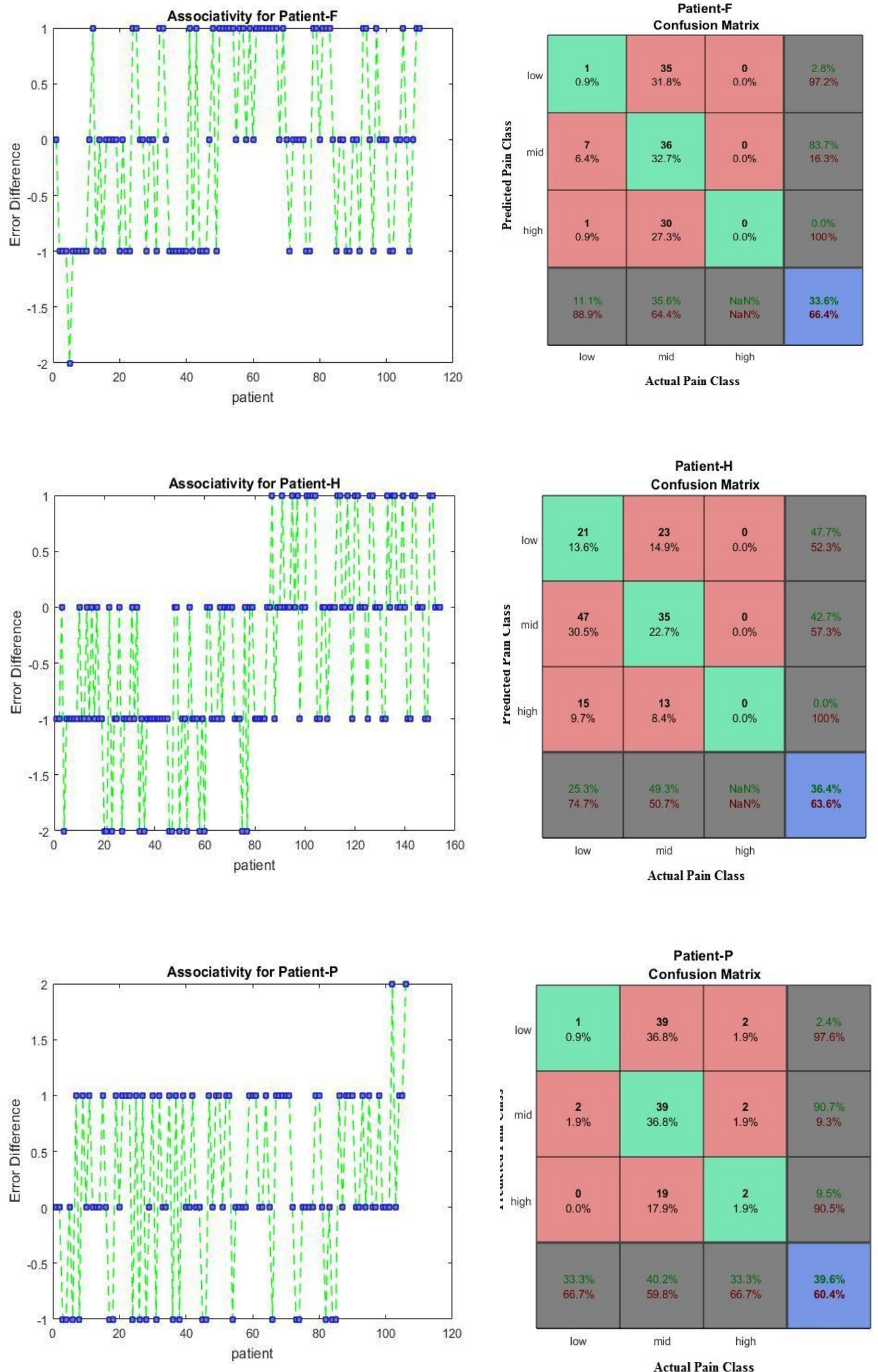

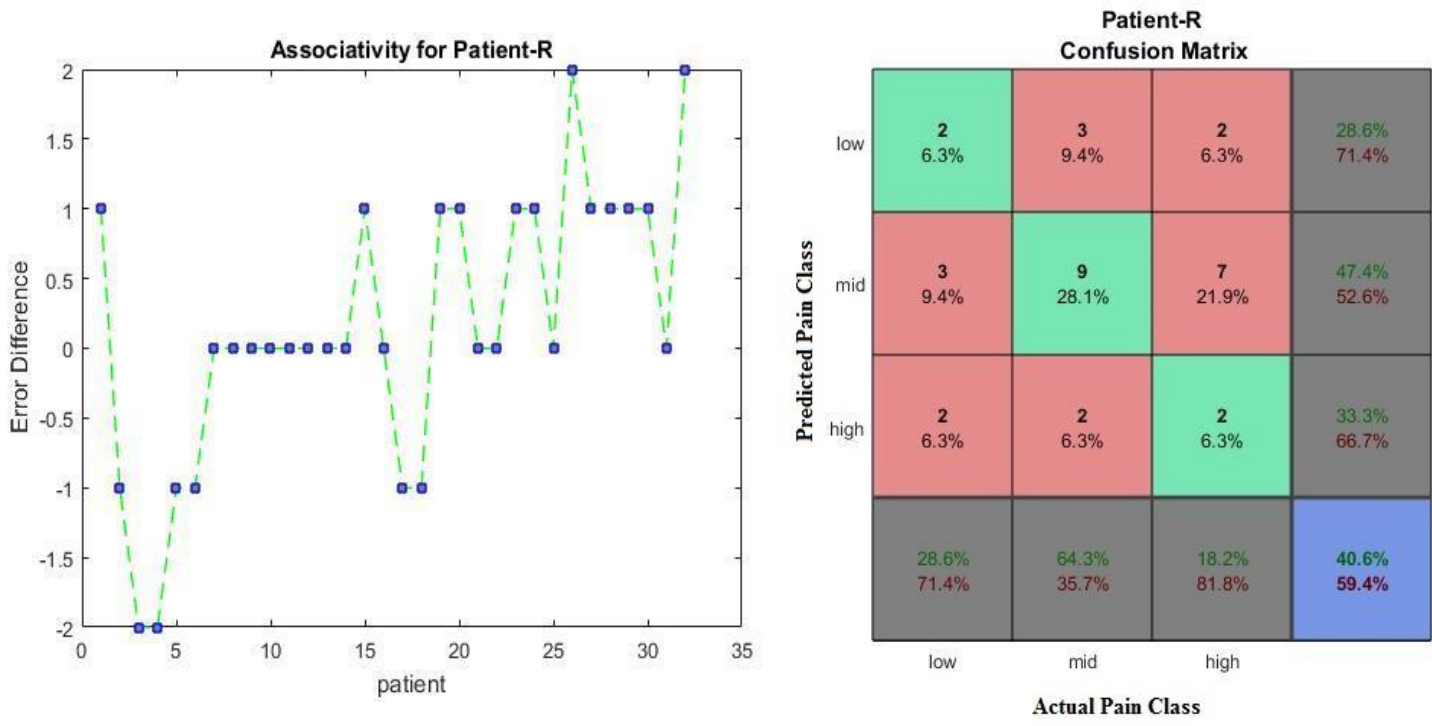

Fig 13: Cross-sectional Evaluation on 4 subjects.

\subsubsection{LONGITUDINAL EVALUATION}

During the longitudinal evaluation of the pain tool, the model was trained with the longitudinal images of the test subject using continuous learning. In continuous learning, when we have the $\mathrm{n}^{\text {th }}$ image, it was tested with the model prepared using the $1 \ldots(n-1)^{\text {th }}$ images. Same transformation into three (3) categorical levels: low (0-3), mid (4-6) and high (7-10) was done like cross-sectional evaluation as described in 8.2.2. Residuals of each level were observed for four (4) subjects. 

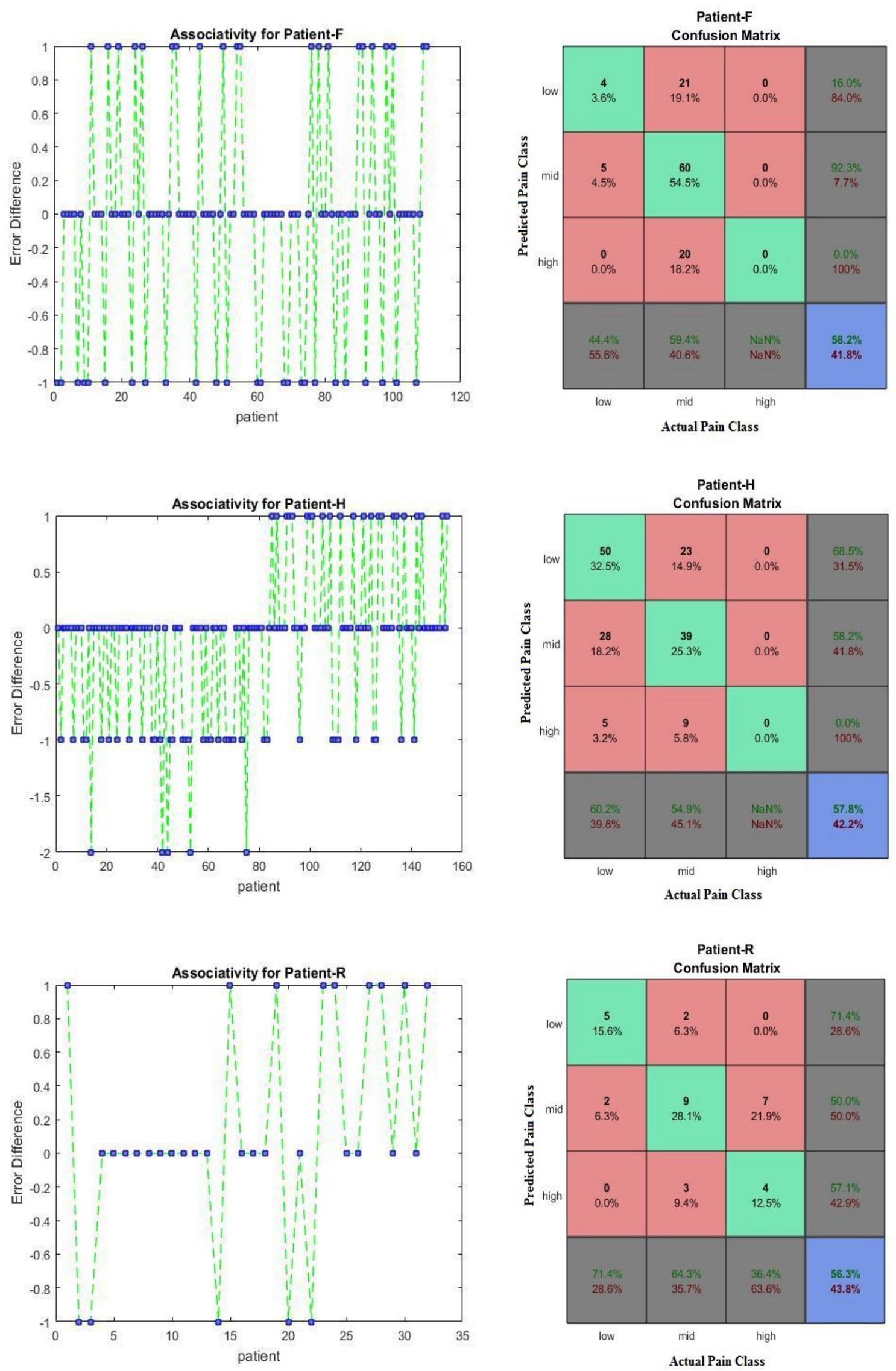

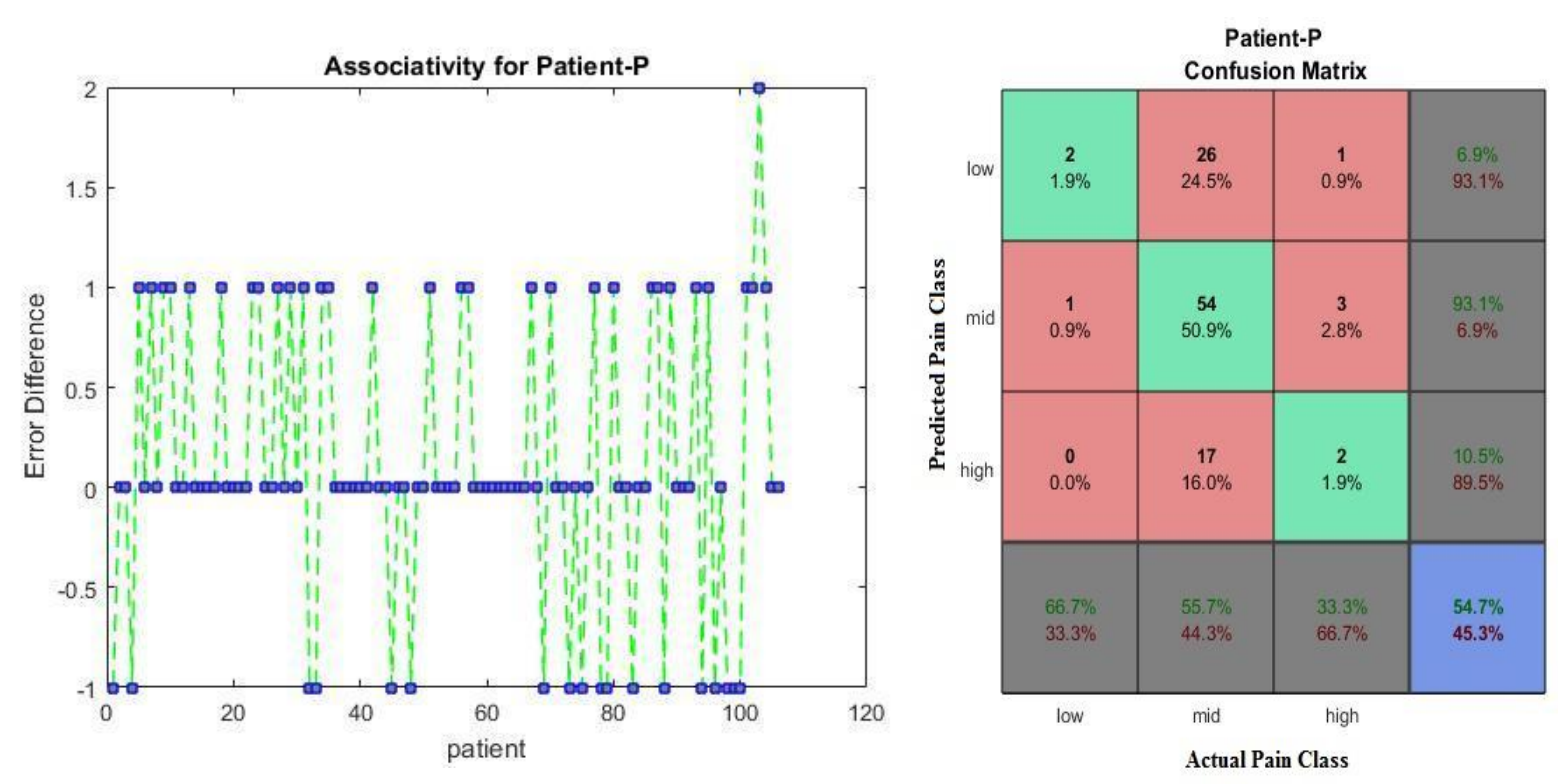

Fig 14: Longitudinal Evaluation on 4 subjects.

\subsubsection{DEMOGRAPHIC EVALUATION}

Demographic evaluation was done on the cross-sectional dataset. In the demographic evaluation, training model was prepared only using cross-sectional images from that specific demography. Due to limited dataset, training and testing dataset was prepared by a randomized $80 \%-20 \%$ ratio. For example, in a evaluation set $80 \%$ of the cross-sectional images from Nepal was used to generate the training model where rest $20 \%$ was used to evaluate the model.

\subsubsection{RESULTS}

The results of the system were evaluated in terms of two performance measure: the mean absolute error and classification accuracy of pain groups. As a consequence of insufficient data for two (2) subjects were not used in the both 
evaluation of the pain tool, the results shown in the Table 6 are only for subject F, H, $\mathrm{P}$ and $\mathrm{R}$.

\begin{tabular}{|c|c|c|c|c|c|c|c|c|}
\hline \multirow{2}{*}{$\begin{array}{c}\text { Cross. } \\
\text { Val }\end{array}$} & \multicolumn{2}{|c|}{ Subject F } & \multicolumn{2}{|c|}{ Subject H } & \multicolumn{2}{|c|}{ Subject P } & \multicolumn{2}{|c|}{ Subject R } \\
\hline & $\mathrm{L}$ & $\mathrm{C}$ & $\mathrm{L}$ & $\mathrm{C}$ & $\mathrm{L}$ & $\mathrm{C}$ & $\mathrm{L}$ & $\mathrm{C}$ \\
\hline 1 & 1.34 & 2.36 & 1.33 & 2.12 & 1.46 & 2.06 & 1.45 & 2.15 \\
\hline 2 & 1.25 & 2.39 & 1.32 & 2.26 & 1.26 & 2.14 & 1.41 & 2.23 \\
\hline 3 & 1.29 & 2.26 & 1.28 & 2.00 & 1.33 & 2.26 & 1.38 & 2.29 \\
\hline 4 & 1.32 & 2.28 & 1.29 & 2.05 & 1.41 & 1.99 & 1.43 & 2.35 \\
\hline 5 & 1.31 & 2.16 & 1.46 & 2.13 & 1.50 & 2.33 & 1.41 & 2.04 \\
\hline 6 & 1.28 & 2.18 & 1.38 & 2.18 & 1.35 & 2.26 & 1.42 & 2.33 \\
\hline 7 & 1.31 & 2.32 & 1.25 & 2.26 & 1.41 & 2.22 & 1.39 & 2.27 \\
\hline 8 & 1.54 & 2.20 & 1.36 & 1.83 & 1.38 & 2.17 & 1.43 & 2.17 \\
\hline 9 & 1.37 & 2.33 & 1.28 & 2.18 & 1.31 & 2.42 & 1.44 & 2.21 \\
\hline 10 & 1.27 & 2.29 & 1.41 & 2.28 & 1.28 & 2.33 & 1.39 & 2.22 \\
\hline Mean & 1.33 & 2.28 & 1.34 & 2.13 & 1.37 & 2.22 & 1.42 & 2.21 \\
\hline $\begin{array}{l}\text { Std. } \\
\text { Dev. }\end{array}$ & 0.08 & 0.08 & 0.07 & 0.14 & 0.08 & 0.13 & 0.02 & 0.09 \\
\hline
\end{tabular}

Table 6: Mean Absolute error for a 10-fold cross validation. 
Mean absolute error is significantly less in continuous learning based longitudinal evaluation with respect to one-time learning based cross-sectional evaluation. The goal is to reduce the mean absolute error is for reproducible usage in clinical settings.

Pain group level classification accuracy is shown in Table 7 for this experiment hereby termed as Experiment-1. It is observed that mid pain level group classification accuracy is better, with mean accuracy of $68 \%$ among the test subjects than other pain group level such as low and high which yields to $50 \%$ and $45 \%$ mean accuracy rate respectively. 


\begin{tabular}{|c|c|c|c|}
\hline Cross Val. & Low-Low & Mid-Mid & High-High \\
\hline 1 & $53 \%$ & $74 \%$ & $50 \%$ \\
\hline 2 & $57 \%$ & $68 \%$ & $47 \%$ \\
\hline 3 & $54 \%$ & $72 \%$ & $39 \%$ \\
\hline 4 & $52 \%$ & $73 \%$ & $45 \%$ \\
\hline 5 & $49 \%$ & $65 \%$ & $52 \%$ \\
\hline 6 & $40 \%$ & $68 \%$ & $41 \%$ \\
\hline 7 & $45 \%$ & $54 \%$ & $46 \%$ \\
\hline 8 & $53 \%$ & $61 \%$ & $42 \%$ \\
\hline 9 & $51 \%$ & $71 \%$ & $40 \%$ \\
\hline 10 & $43 \%$ & $69 \%$ & $51 \%$ \\
\hline Mean & $50 \%$ & $68 \%$ & $45 \%$ \\
\hline Std. Dev. & 0.05 & 0.06 & 0.04 \\
\hline
\end{tabular}

Table 7: Classification Accuracy of Experiment-1 for 10-fold cross validation.

Better classification accuracy can be attributed to higher training data in the dataset. For experiment-1, based on pain level category definition mid and high pain category are the highest and lowest class with $70.3 \%$ and $4.2 \%$ respectively. Class distribution in the longitudinal dataset is shown below in Figure 15. 


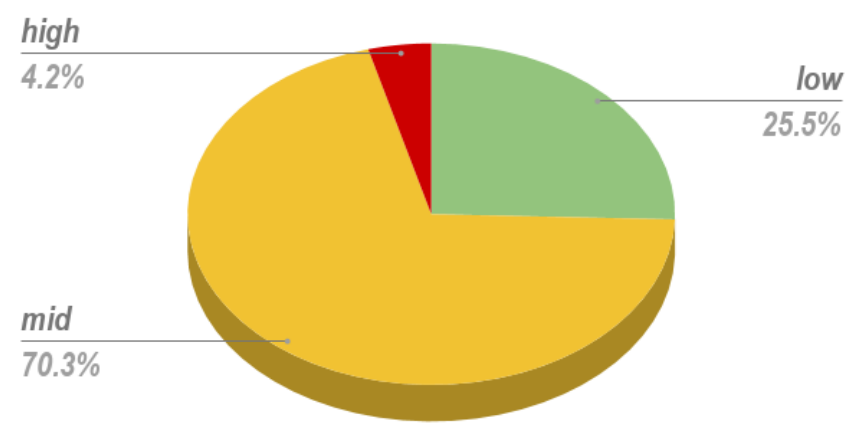

Fig 15: Class-distribution for Experiment -1 in Longitudinal Dataset

The cross-sectional demographic evaluation on the cross-sectional dataset also conforms to the relation between better classification accuracy and size of training dataset.

\begin{tabular}{|c|c|c|c|c|}
\hline \multirow{2}{*}{ Location } & \multicolumn{3}{|c|}{ 10-fold Mean Accuracy } & \multirow{2}{*}{ Population } \\
\cline { 2 - 4 } & Low-Low & Mid-Mid & High-High & \\
\hline Bangladesh & $53 \%$ & $73 \%$ & $48 \%$ & 743 \\
\hline Nepal & $51 \%$ & $62 \%$ & $35 \%$ & 620 \\
\hline South Dakota & $26 \%$ & $37 \%$ & $17 \%$ & 80 \\
\hline Mean & $43 \%$ & $57 \%$ & $33 \%$ & \\
\hline Std. Dev. & 0.15 & 0.18 & 0.16 & \\
\hline
\end{tabular}

Table 8: Cross-sectional Demographic Evaluation

In order to further validate the prediction accuracy behavior with respect to training data set size, Experiment -2 was conducted with more flexible constraint on categories of pain levels. For Experiement-2, with continuous learning model pain levels were categorized to same three (3) categorical levels but with different pain ranges: low (0-2), mid (3-7) and high (8-10). This experiment with flexible constraints 
resulted in a slightly improved result for mid-level pain group but slightly down accuracy in low-level pain group. Findings of Experiment-2 are shown in following Table-8.

\begin{tabular}{|c|c|c|c|}
\hline Cross Val. & Low-Low & Mid-Mid & High-High \\
\hline 1 & $42 \%$ & $69 \%$ & $46 \%$ \\
\hline 2 & $47 \%$ & $78 \%$ & $43 \%$ \\
\hline 3 & $38 \%$ & $72 \%$ & $34 \%$ \\
\hline 4 & $39 \%$ & $62 \%$ & $45 \%$ \\
\hline 5 & $39 \%$ & $65 \%$ & $49 \%$ \\
\hline 6 & $44 \%$ & $68 \%$ & $41 \%$ \\
\hline 7 & $45 \%$ & $79 \%$ & $42 \%$ \\
\hline 8 & $48 \%$ & $67 \%$ & $42 \%$ \\
\hline 9 & $39 \%$ & $74 \%$ & $39 \%$ \\
\hline 10 & $42 \%$ & $73 \%$ & $51 \%$ \\
\hline Mean & $42 \%$ & $71 \%$ & $43 \%$ \\
\hline Std. Dev. & $42 \%$ & $69 \%$ & $46 \%$ \\
\hline
\end{tabular}

Table 9: Classification Accuracy of Experiment-2 for 10-fold Cross Validation. 
For the experiment-2, based on our new flexible pain level category definition mid is still the highest class with $89.5 \%$ whereas the lowest class is high with $1.0 \%$ of the dataset. Class distribution in the longitudinal dataset for Experiment-2 is shown below in Figure 16.

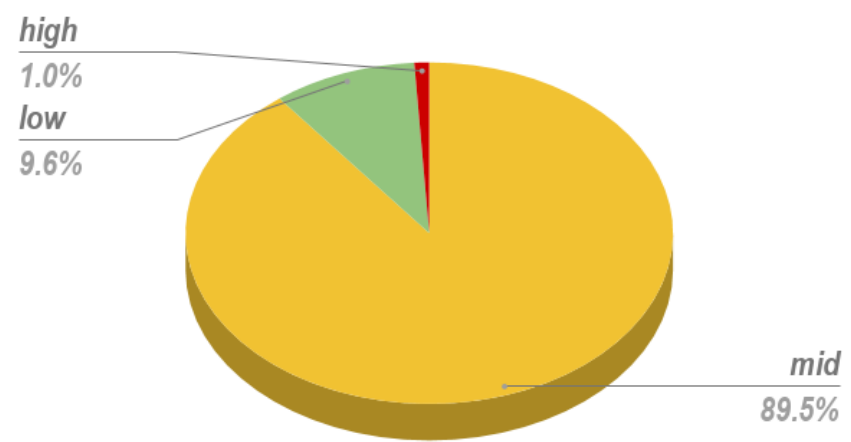

Fig 16: Class-distribution for Experiment -2 in Longitudinal Dataset 


\section{CHAPTER 8}

EVALUATION

\subsection{SUMMARY}

In this thesis, we have proposed, designed and developed a pain study platform along with a facial image based automated pain tool which facilitates researchers and participants to with more collaboration opportunity. The goal of the platform is to enhance machine learning based capabilities for researchers and clinicians in diagnosing, designing and evaluating interventions for pain patients.

\subsection{INTELLECTUAL MERIT}

For this thesis, we have a contribution in developing a first-time pain data collaboration platform specifically designed for pain studies, real -time data collection, multiple research collaboration on the same data and/or participants, an improved automatic pain detection tool which yields promising results. This software research platform is responsive and HIPAA compliant which makes it easier to deploy to participants and privacy aware and secured.

\subsection{BROADER IMPACT}

By bringing healthcare services to the remote users, mobile communication and smart technology devices are helping to improve the life quality. It is very common to find research studies which collect data of participants and thus help to 
improve participants' health using that data. But these data collections for research studies are generally performed in a nucleus manner which makes it harder to use for any future researches. To best of our knowledge there is no platform which is device independent and generic, offers collaborative interfaces for both pain researcher and pain patient participant, collects data electronically, provides researcher real time accessible data and most significantly which stores user information in a secured and privacy-aware manner. We have designed and developed our pain study platform which addresses all these areas.

\subsection{FUTURE WORKS}

Automatic pain detection from facial images is a challenging research problem. Long term context aware data collection and testing different algorithms for differently scoped pain study is a future work for this pain detection tool. Long term usability testing of the platform will also help the platform to identify and incorporate useful functionality features. 


\section{BIBLIOGRAPHY}

[1] G. Lu, C. Yang, M. Chen and X. Li, "Sparse representation based facial expression classification for pain assessment in neonates," 2016 12th International Conference on Natural Computation, Fuzzy Systems and Knowledge Discovery (ICNC-FSKD), Changsha, 2016, pp. 1615-1619.

doi: 10.1109/FSKD.2016.7603418

keywords: \{emotion recognition;medical image processing;paediatrics;principal component analysis;Principal Component Analysis;computer based image analysis;facial expression classification;neonatal pain assessment;neonates;sparse representation;weighted Local Binary Pattern;Face;Face recognition;Feature extraction;Histograms;Pain;Pediatrics;Training;expression recognition;local binary pattern;neonate;pain assessment;sparse representation \},

URL:

http://ieeexplore.ieee.org/stamp/stamp.jsp?tp=\&arnumber=7603418\&isnumber $=7603$ $\underline{138}$

[2] D. L. Martinez, O. Rudovic and R. Picard, "Personalized Automatic Estimation of Self-Reported Pain Intensity from Facial Expressions," 2017 IEEE Conference on Computer Vision and Pattern Recognition Workshops (CVPRW), Honolulu, HI, 2017, pp. 2318-2327.

doi: 10.1109/CVPRW.2017.286

keywords: \{face recognition;learning (artificial intelligence);medical computing;recurrent neural nets;HCRF;PSPI;Prkachin and Solomon pain intensity levels;RNN;VAS;automatic pain detection systems;face images;facial expressions;facial expressiveness score;pain analysis;pain score acquisition efforts;personalized automatic estimation;personalized hidden conditional random fields;recurrent neural networks;self-reported pain intensity;subjective experience;two-stage learning approach;visual analog scales;Estimation;Face;Hidden Markov models;Image sequences;Pain;Recurrent neural networks;Reliability\},

URL:

http://ieeexplore.ieee.org/stamp/stamp.jsp?tp=\&arnumber=8015020\&isnumber $=8014$ $\underline{734}$

[3] Bellantonio M. et al. (2017) Spatio-temporal Pain Recognition in CNN-Based Super-Resolved Facial Images. In: Nasrollahi K. et al. (eds) Video Analytics. Face and Facial Expression Recognition and Audience Measurement. FFER 2016, VAAM 2016. Lecture Notes in Computer Science, vol 10165. Springer, Cham URL: https://link.springer.com/chapter/10.1007/978-3-319-56687-0_13

[4] F. Wang et al., "Regularizing face verification nets for pain intensity regression," 2017 IEEE International Conference on Image Processing (ICIP), Beijing, 2017, pp. 1087-1091. doi: 10.1109/ICIP.2017.8296449 keywords: \{emotion recognition;face recognition;feature extraction;image representation;image sequences;learning (artificial intelligence);regression analysis;face verification nets;pain intensity regression;facial expression intensities;deep networks;automated pain 
assessment;patient-reported pain intensities;regularized regression loss;UNBCMcMaster Shoulder-Pain dataset;pain expression intensity;regularized deep regressor;rich feature representations; expression intensity regression task; expression labels;Pain;Face;Convolution;Training;Distance measurement;Biomedical monitoring;fine-tuning;CNN;regularizer;regression\},

URL:

http://ieeexplore.ieee.org/stamp/stamp.jsp?tp=\&arnumber $=8296449 \&$ isnumber $=8296$ $\underline{222}$

[5] Patrick Lucey, Jeffrey F Cohn, Kenneth M Prkachin, Patricia E Solomon, and Iain Matthews, "Painful data: The unbc-mcmaster shoulder pain expression archive database," in Proceedings of the IEEE International Conference on Automatic Face \& Gesture Recognition, 2011, pp. 57-64.

[6] Min Aung, Sebastian Kaltwang, Bernardino RomeraParedes, Brais Martinez, Aneesha Singh, Matteo Cella, Michel Valstar, Hongying Meng, Andrew Kemp, Aaron Elkins, et al., "The automatic detection of chronic painrelated expression: requirements, challenges and a multimodal dataset," IEEE Transactions on Affective Computing, vol. 7, no. 4, pp. 435-451, 2016.

[7] Philipp Werner, Ayoub Al-Hamadi, Robert Niese, Steffen Walter, Sascha Gruss, and Harald C Traue, "Towards pain monitoring: Facial expression, head pose, a new database, an automatic system and remaining challenges," in Proceedings of the British Machine Vision Conference, 2013

[8] Machine-based Multimodal Pain Assessment Tool for Infants: A Review Ghada Zamzmi, Chih-Yun Pai, Dmitry Goldgof, Rangachar Kasturi, Yu Sun, Terri Ashmeade. URL: https://arxiv.org/abs/1607.00331

[9] Neeru Rathee, Dinesh Ganotra (2017) A Novel Approach for Continuous Pain Intensity Estimation. In: Singh R., Choudhury S. (eds) Proceeding of International Conference on Intelligent Communication, Control and Devices. Advances in Intelligent Systems and Computing, vol 479. Springer, Singapore

[10] Hasan, Md Kamrul \& Ahsan, Golam Mushih Tanimul \& Ahamed, Sheikh \& Love, Rechard \& Salim, Reza. (2016). Pain Level Detection From Facial Image Captured by Smartphone. Journal of Information Processing. 24. 598-608. 10.2197/ipsjjip.24.598.

[11] Alberto Marcial Basilio, Jorge \& Aguilar Torres, Gualberto \& Sanchez-Perez, Gabriel \& Karina Toscano Medina, L \& Perez-Meana, Hector. (2011). Explicit image detection using YCbCr space color model as skin detection. 123-128. 
[12] Fausto Giunchiglia, Mattia Zeni, Elisa Gobbi, Enrico Bignotti, Ivano Bison, Mobile social media usage and academic performance, Computers in Human Behavior, Volume 82,2018, Pages 177-185,ISSN 07475632,https://doi.org/10.1016/j.chb.2017.12.041.

(http://www.sciencedirect.com/science/article/pii/S0747563217307276)

Keywords: Social media; Academic performance; Smartphone; Time diaries

[13] MEASURING POWER CONSUMPTION FOR IMAGE PROCESSING ON ANDROID SMARTPHONE, Khairul Muzzammil Saipullah, Ammar Anuar, Nurul Atiqah Ismail and Yewguan Soo. American Journal of Applied Sciences, 2012, 9 (12), 2052-2057 ISSN: 1546-9239 (C2012 Science Publication doi:10.3844/ajassp.2012.2052.2057 Published Online 9 (12) 2012 (http://www.thescipub.com/ajas.toc)

[14] K. Kumar and Y. H. Lu, "Cloud Computing for Mobile Users: Can Offloading Computation Save Energy?," in Computer, vol. 43, no. 4, pp. 51-56, April 2010. doi: 10.1109/MC.2010.98

keywords: \{Internet;energy conservation;mobile computing;Internet;application services;battery lifetimes;cloud computing;computation offloading;mobile systems;mobile users;Batteries;Cloud computing;Computer applications;Mobile computing; Web and internet services;Cloud computing;Energy savings;Mobile/wireless \},

URL:

http://ieeexplore.ieee.org/stamp/stamp.jsp?tp=\&arnumber=5445167\&isnumber $=5445$ $\underline{153}$

[15] Application development and extensibility/customization using entity modeling systems and methods. https://patents.google.com/patent/US20170322783A1/en

[16] https://gupea.ub.gu.se/bitstream/2077/20561/1/gupea_2077_20561_1.pdf

[17] Martin, Robert (2002). Agile Software Development: Principles, Patterns and Practices. Pearson Education.

[18] "Best Practices for Software Development Projects". IBM.

Date Accessed 05/29/2016

URL:

http://www.ibm.com/developerworks/websphere/library/techarticles/0306_perks/perk $\underline{\text { s2.html }}$

[19] “Which Life Cycle is Best for Your Project?". Project Smart. Date Accessed $04 / 10 / 2018$ 
URL: https://www.projectsmart.co.uk/which-life-cycle-is-best-for-your-project.php [20] Smith, A. K., Ayanian, J. Z., Covinsky, K. E., Landon, B. E., McCarthy, E. P., Wee, C. C., \& Steinman, M. A. (2011). Conducting High-Value Secondary Dataset Analysis: An Introductory Guide and Resources. Journal of General Internal Medicine, 26(8), 920-929. http://doi.org/10.1007/s11606-010-1621-5

[21] "Why Continuous Learning is the key towards Machine Intelligence", Date Accessed 04/16/2018 URL:https://medium.com/@vlomonaco/why-continuouslearning-is-the-key-towards-machine-intelligence-1851cb57c308

[22] P. Lucey, J. F. Cohn, K. M. Prkachin, P. E. Solomon and I. Matthews, "Painful data: The UNBC-McMaster shoulder pain expression archive database," Face and Gesture 2011, Santa Barbara, CA, 2011, pp. 57-64. doi: 10.1109/FG.2011.5771462 keywords: \{emotion recognition;face recognition;image coding;image representation;image sequences;psychology;support vector machines;visual databases;AAM-SVM system;FACS coded frame;McMaster University;UNBCMcMaster shoulder pain expression archive database;University of Northern British Columbia;associated pain frame-by-frame score;automatic pain detection;data distribution;facial expression;functional automatic facial expression detection system;observer measure;painful data;passive range-of-motion test;patient face representation;target application; unaffected limb; video sequence;Active appearance model;Databases;Face;Gold;Observers;Pain;Shape \},

URL:

http://ieeexplore.ieee.org/stamp/stamp.jsp?tp=\&arnumber=5771462\&isnumber $=5771$ $\underline{322}$

[23] W. A. Shier and S. Yanushkevich, "Pain recognition and intensity classification using facial expressions," 2016 International Joint Conference on Neural Networks (IJCNN), Vancouver, BC, 2016, pp. 3578-3583.

doi: 10.1109/IJCNN.2016.7727659 keywords: \{Gabor filters;biometrics (access control);face recognition;feature extraction;image classification;support vector machines;Gabor energy filter;Prkachin pain intensity scale;Solomon pain intensity scale;facial biometrics;facial expression analysis;intensity classification;no pain;pain classification;pain detection;pain recognition;strong pain;support vector machines;weak pain;Emotion recognition;Face;Face recognition;Feature extraction;Pain;Support vector machines;Training\},

URL:

http://ieeexplore.ieee.org/stamp/stamp.jsp?tp=\&arnumber=7727659\&isnumber $=7726$ 591

[24] Wewers, M. E., \& Lowe, N. K. (1990). A critical review of visual analogue scales in the measurement of clinical phenomena. Research in Nursing \& Health, 13(4), 227-236.

http://dx.doi.org/10.1002/nur.4770130405 
[25] Hawker, G. A., Mian, S. , Kendzerska, T. and French, M. (2011), Measures of adult pain: Visual Analog Scale for Pain (VAS Pain), Numeric Rating Scale for Pain (NRS Pain), McGill Pain Questionnaire (MPQ), Short-Form McGill Pain Questionnaire (SF-MPQ), Chronic Pain Grade Scale (CPGS), Short Form-36 Bodily Pain Scale (SF-36 BPS), and Measure of Intermittent and Constant Osteoarthritis Pain (ICOAP). Arthritis Care Res, 63: S240-S252. doi:10.1002/acr.20543

[26] Huskisson EC. Measurement of pain. Lancet 1974; 2: 1127-31.

[27] Joyce CR, Zutshi DW, Hrubes VF, Mason RM. Comparison of fixed interval and visual analogue scales for rating chronic pain. Eur J Clin Pharmacol 1975; 8: 415-20.

[28] Monwar, M., Rezait, S. and Prkachin, K.: Eigenimage Based Pain Expression Recognition, IAENG International Journal of Applied Mathmatics, Vol.36, No.2 (2006)

[29] Adibuzzaman, Mohammad \& Ostberg, Colin \& Ahamed, Sheikh \& Povinelli, Richard \& Sindhu, Bhagwant \& Love, Richard \& Kawsar, Ferdaus \& Ahsan, Golam Mushih Tanimul. (2015). Assessment of Pain Using Facial Pictures Taken with a Smartphone. 726-731. 10.1109/COMPSAC.2015.150.

[30] Database Systems: Design, Implementation, \& Management By Carlos Coronel, Steven Morris. Course Technology; 10 edition (January 1, 2012).

[31] C. M. Spies, "Proposed model for evaluation of mHealth systems," 2015 International Conference on Computing, Communication and Security (ICCCS), Pamplemousses, 2015, pp. 1-8. doi: 10.1109/CCCS.2015.7374199

keywords: \{health care;mobile computing;mhealth system;mobile health;system evaluation;Computers;Conferences;MIMO;Robustness;State feedback;Transfer functions;Yttrium;mHealth;system design;system evaluation\}, URL:

http://ieeexplore.ieee.org/stamp/stamp.jsp?tp=\&arnumber=7374199\&isnumber $=7374$ $\underline{113}$

[32] “Usability in Software Design”. Microsoft. Date Accessed 04/27/2018 https://msdn.microsoft.com/en-us/library/ms997577.aspx 
[33] Daihua X. Yu et al."Accessible mHealth for patients with dexterity impairments" Proceeding ASSETS '14 Proceedings of the 16th international ACM SIGACCESS conference on Computers \& accessibility. Pages 235-236. doi>10.1145/2661334.2661402 URL: https://dl.acm.org/citation.cfm?id=2661402 [34] An investigation of user interface design and development of a mobile health system for individuals with dexterity impairments

Yu, Daihua Xie. University of Pittsburgh, ProQuest Dissertations Publishing, 2014. 3690875.

[35] R. Patel, W. Green, M. W. Shahzad, and C. Larkin, "Use of Mobile Clinical Decision Support Software by Junior Doctors at a UK Teaching Hospital: Identification and Evaluation of Barriers to Engagement," JMIR mHealth and uHealth, vol. 3, no. 3, p. e80, 2015.

[36] I. Kim, P.-H. Lai, R. Lobo, and B. J. Gluckman, "Challenges in wearable personal health monitoring systems," in Engineering in Medicine and Biology Society (EMBC), 2014 36th Annual International Conference of the IEEE, 2014, pp. 52645267.

[37] M. Ji, Y. Wu, P. Chang, X. Yang, F. Yang, and S. Xu, "Development

and Usability Evaluation of the Mobile Delirium Assessment App Based on Confusion Assessment Method for Intensive Care Unit (CAM-ICU).," Studies in health technology and informatics, vol. 216, pp. 899-899, 2014.

[38] E. Jovanov, "Wireless Technology and System Integration in Body Area Networks for m-Health Applications.," Conf Proc IEEE Eng Med Biol Soc, vol. 7, pp. 7158-60, 2005.

[39] Jane Katusiime and Niels Pinkwart, “A review of privacy and usability issues in mobile health systems: Role of external factors", Health Informatics Journal, https://doi.org/10.1177/1460458217733121

[40] Kainda R, Fléchais I and Roscoe AW.Security and usability: analysis and evaluation. In: 2010 international conference on availability, reliability and security, Krakow, 15-18 February 2010, pp. 275-282. New York: IEEE.

[41] Ferraiolo, D.F. \& Kuhn, D.R. (October 1992). "Role-Based Access Control" (PDF). 15th National Computer Security Conference: 554-563.

[42] Sandhu, R., Coyne, E.J., Feinstein, H.L. and Youman, C.E. (August 1996). "Role-Based Access Control Models" (PDF). IEEE Computer. IEEE Press. 29 (2): 38-47. doi:10.1109/2.485845. 
[43] A.C. O'Connor \& R.J. Loomis (March 2002). Economic Analysis of Role-Based Access Control (PDF). Research Triangle Institute.

[44] "The HIPAA Security Rule". U.S. Department of Health and human Services.

Date Accessed 05/03/2018, URL: http://www.hhs.gov/hipaa/forprofessionals/security/

[45] "The HIPAA Privacy Rule". U.S. Department of Health and human Services.

Date Accessed 05/03/2018, URL: http://www.hhs.gov/hipaa/forprofessionals/privacy/

[46] "How To Perfect Your Mobile App's Login Screen”, UsabilityGeek, Approved Educational Partner of the Interaction Design Foundation (IDF) - the world's largest UX Design learning community.

Date Accessed 05/03/2018. URL: https://usabilitygeek.com/perfect-mobile-app-loginscreen/

[47] "PHP". Wikipedia. Date Accessed 05/04/2018

URL: https://en.wikipedia.org/wiki/PHP

[48] “About Yii”. Yii 2 Guide. Date Accessed 05/04/2018.

URL: http://www.yiiframework.com/doc-2.0/guide-intro-yii.html

[49] "Bootstrap in A List Apart No. 342". Otto, Mark (17 January 2012). Date Accessed 04/13/2018

URL: http://markdotto.com/2012/01/17/bootstrap-in-a-list-apart-342/

[50] Doaa Nabil, Abeer Mosad, Hesham A. Hefny, Web-Based Applications quality factors: A survey and a proposed conceptual model, Egyptian Informatics Journal, Volume 12, Issue 3, 2011, Pages 211-217, ISSN 1110-8665, https://doi.org/10.1016/j.eij.2011.09.003.

(http://www.sciencedirect.com/science/article/pii/S1110866511000405)

Keywords: Web Based Applications; Quality; Quality assurance; Quality models

[51] ISO/IEC 25010:2011. Systems and software engineering -- Systems and software Quality Requirements and Evaluation (SQuaRE) -- System and software quality models by Joint Technical Committee ISO/IEC JTC 1, Information technology, Subcommittee SC 7, Software and systems engineering.

[52] "ISO/IEC 25010", Date Accessed 04/31/20018

URL: http://iso25000.com/index.php/en/iso-25000-standards/iso-25010 